\title{
Surface Nanobubbles: Theory, Simulation, and Experiment. A Review
}

\author{
Panagiotis E. Theodorakis ${ }^{\mathrm{a}, *}$, Zhizhao Che $\mathrm{e}^{\mathrm{b}, * *}$ \\ ${ }^{a}$ Institute of Physics, Polish Academy of Sciences, Al. Lotników 32/46, 02-668 Warsaw, \\ Poland \\ ${ }^{b}$ State Key Laboratory of Engines, Tianjin University, Tianjin, 300072, China
}

\begin{abstract}
Surface nanobubbles (NBs) are stable gaseous phases in liquids that form at the interface with solid substrates. They have been particularly intriguing for their high stability that contradicts theoretical expectations and their potential relevance for many technological applications. Here, we present the current state of the art in this research area by discussing and contrasting main results obtained from theory, simulation and experiment, and presenting their limitations. We also provide future perspectives anticipating that this review will stimulate further studies in the research area of surface NBs.

Keywords: Surface Nanobubbles, Experiment, Theory, Simulation, Contact-line Pinning, Oversaturation
\end{abstract}

\section{Introduction}

Surface nanobubbles (NBs) are gaseous domains at the interface between a liquid medium (e.g. water) and a solid substrate, which is usually hydrophobic (see Fig. 1] 14 4. The existence of NBs was speculated about 25 years ago by Parker et al. as they attempted to estimate the forces between two neutral hydrophobic surfaces immersed in water [5]. In this case, discontinuities in the force measurements were attributed to microscopic bubbles or cavities between the surfaces. By carrying out Atomic Force Microscopy (AFM) measurements,

* panos@ifpan.edu.pl

Preprint submitted to Advances in Colloid and Interface Science

September 9, 2019 
Carambassis et al. later suggested that a long-range attraction between the immersed surfaces was due to long-lived submicron bubbles [6]. Subsequent investigations have drawn similar conclusions in the case of hydrophobised silica surfaces providing initial evidence of NBs existence [7, 8].

Despite these early studies, the existence of stable surface NBs was established by Lou et al., who provided the first AFM image of hour-long stable NBs on a mica surface immersed in water [9. The acquisition of AFM images was possible due to the apparently slower relaxation of the NBs in comparison with the motion of the AFM tip, which could sense a repulsive force during the interaction with the NBs. In the same year, images of surface NBs on silicon wafer hydrophobised with octadecyltrichlorosilane (OTS) were obtained by Ishida et al., providing further evidence for the existence of surface NBs [10]. These early studies indicated that the shape of NBs resembled roughly a spherical cap with height ranging from one to tens of nanometres and base radius from hundreds of nanometres to several microns. Moreover, the apparent contact angle (measured on the gas side, see Fig. 1) of the NBs was much smaller than the macroscopically expected values [10]. Further experiments suggested that the contact angle be also a function of NB size [9, 11 14, while their density be temperature-dependent [15]. Work by Tyrrell et al. by means of AFM experiments indicated that the radius of curvature of the NBs was of the order of $100 \mathrm{~nm}$, while the height was 20-30 nm [12. This particular morphology of surface NBs was believed to be the reason for their unexpected high stability [10, 12] despite the theoretically predicted high Laplace pressure $(\Delta p=2 \gamma / R$, where $\gamma$ is the surface tension and $R$ the bubble radius, see Fig. 11 [16, which would suggest their fast dissolution in a time scale of a few microseconds [14, 17. Yet, the stability of surface NBs has been confirmed on different substrates, for example, in the case of Highly Oriented Pyrolytic Graphite (HOPG) substrates [18, 19] and ultraflat gold surfaces [20, while unstable surface NBs have been reported in the case of HOPG substrates immersed in alcohol [21, 22.

The existence of surface NBs has been further established by different experiments, such as Attenuated Total Reflectance Infrared Spectroscopy [23, 
24, Quartz Crystal Microbalance [25, 26], Surface Plasmon Resonance [23, 24, 27, and Neutron Reflectometry [28. In the case of single NBs, the evidence stems from Scanning Electron Microscopy (SEM) imaging [29, Scanning Transmission Soft X-ray Microscopy [30], and Single Photon Counting combined with Fluorescence Lifetime Imaging Microscopy [31]. Direct observation with Interference-Enhanced Reflection Microscopy [32] and with Total-InternalReflection-Fluorescence Microscopy (TIRF) has been also recently provided in the literature 33 35.

Owing to their high stability and spontaneous formation, surface NBs are important in the context of many modern applications where the role of surface interactions becomes relevant [36, 37, such as separation processes 38. Surface NBs have been also found to play a significant role in the case of flotation [39, drag reduction [40, 41], nanocomposite foams [42, 43], nanomaterial engineering [44, catalysis and electrolysis [45], plasmonic and vapour NBs [46], and transport in nanofluidic devices as well as boiling heat transfer by serving as nucleation sites [4]. The fabrication of nanoporous polypyrrole (PPy) films is a specific example, where stable NBs can be used as a template to create nanoporous films [48. In this case, hydrogen surface NBs electrochemically form on a bare HOPG substrate. Then, electropolymerisation of pyrrole takes place around the surface NBs. In the end, PPy nanoporous films are obtained by removing the NBs.

The subsequent sections of this review article are organised as follows: In Sec. 2, we present a range of different theoretical, simulation and experimental methodologies, which are used to study surface NBs discussing their limitations and contrasting main results. In Sec. 3. we discuss the main morphological and mechanical characteristics of surface NBs. In Sec. 4. we provide a review of the main experimental methods to generate surface NBs. In Sec. 5 we discuss the main hypothesis that supports the intriguing high NBs' stability, i.e. contactline pinning. Finally, we present our perspectives in the research area of surface NBs in Sec. 6. 


\section{Methods to study NBs and main results}

This part of the review describes different methodologies for studying NBs and main results obtained by theoretical (Section 2.1), molecular dynamics simulation (Section 2.2), and experimental studies (Section 2.3). We contrast these results and discuss the limitations of the various methods.

\subsection{Theory}

The rate of dissolution by diffusion of a gas (bulk) bubble without translational motion in an undersaturated solution as well as the rate of bubble growth in an oversaturated solution may be described by the Epstein-Plesset theory [16. While both solutions (either for the growth or the dissolution process) are similar, the rate of bubble growth is inversely proportional to the radius of the bubble and the gas diffusivity. Considering estimates for bubble size in the range 10-100 nm, an extension of the Epstein-Plesset theory would suggest that the bubbles have short lifetimes [49]. Aiming at explaining the high stability of surface NBs, many theories have been proposed to fill the gap in our understanding, such as the contamination at the gas-water interface that hinders the gas exchange and reduces the surface tension [50], the dynamic equilibrium [51] that postulates that the NBs' stability is due to a balance between gas outflux and influx, and the pinning of the contact line [30, 51,55]. Weijs and Lohse [52], have initially considered the NB as a source of gas oversaturation, which dissipates by diffusion as the liquid equilibrates to saturation. Later, Lohse and Zhang [55] and Chan et al. [35] have assumed only gas transport in the vicinity of the NB. The latter two approaches have been recently coupled into a theoretical model, which can explain the stability and dynamics of surface NBs in undersaturated environments [56]. The framework of the model of Lohse and Zhang [55] has been recently exploited to describe the coarsening process of competitively growing NBs that interact diffusively [57]. They found that the coarsening slows down with advancing time and increasing bubble distance.

Contamination theory has attempted to describe the unique properties of surface NBs (i.e. the low contact angle as measured from the gas side, see 
Fig. 1. and the high stability) by suggesting that a film of contaminants would form at the liquid-gas interface, which may be responsible for a reduction of the surface tension that could eventually lead to the dissociation of bubbles [50]. In turn, this would allow for a low gas-side contact angle of the NBs (Fig. 1) and a higher diffusion of gas outside NBs, which would lead to a higher stability. This hypothesis has been tested quantitatively by Das et al. [58, who estimated the dependence of the contact angle and the Laplace pressure on the fraction of impurity coverage at the liquid-gas interface. According to this estimate, the increase in the fractional coverage of the impurities leads to the reduction of both the contact angle and the Laplace pressure. An extension of this model can account for further nonidealities at the liquid-gas interface [59]. Such nonidealities can predict a significantly lower contact angle and Laplace pressure even at a smaller impurity coverage, which highlights further the role of impurities in the stability of NBs. Another extension refers to the consideration of surfactants as 'impurities' by Zhang et al. 60. In this case, water-soluble surfactants appeared to have little effect on the stability of NBs, whereas water-insoluble surfactants dramatically reduced their formation and stability. However, Zhang et al. suggest that NBs stability could not be attributed to a layer of organic contaminants originating from the HOPG or other surface-active materials, for example, as a result of the standard solvent exchange method for generating NBs [60].

Dynamic equilibrium is another theory to explain the stability of surface NBs. It suggests that a continuous influx of gas near the contact line be able to sustain and stabilise the NBs gas phase in contact with hydrophobic substrates (see Fig. 2B) [51]. According to this theoretical model, the substrate hydrophobicity leads to the attraction between the gas and the substrate, which results in a gas influx that balances an expected outflux due to the Laplace pressure, in this way establishing a dynamic equilibrium [23, 61. The model also predicts the equilibrium radius of the NBs and a threshold for gas concentration and substrate hydrophobicity that can enable such dynamic equilibrium [51]. In particular, the stability of NBs is observed for a narrow temperature and 
dissolved-gas concentration range 62. Moreover, it has been suggested that NBs are of Knudsen type in order to be stable in view of the thermal energy of the substrate and the diffusive nature of the liquid-gas interface (see Fig. $2 \mathrm{C}$ 63. Due to the gas flow, a related liquid flow as a circulatory stream from the apex to the three-phase contact line would manifest itself and would further guarantee the balance between gas influx and outflux, in agreement with experimental observations 62. The dynamic equilibrium model has predicted an upper bound for the NB size, which is also in agreement with experiments 62. To this end, the maximum and minimum bubble size decreases with temperature 64, while the gas-side contact angle has been found to decrease with decreasing NB size 65, 66.

Finally, classical Density Functional Theory (DFT) as a numerical and theoretical method has contributed to a better understanding of the thermodynamic properties of surface NBs. In particular, constrained Lattice Density Functional Theory (LDFT) and kinetic LDFT suggest that NBs exist in a thermodynamic metastable state rather than at a thermodynamic equilibrium [67, in agreement with recent simulation studies 68. DFT could readily provide relationships for the gas-side contact angle, size, and chemical potential of NBs, suggesting that NBs stability stems from contact line pinning, which is a result of oversaturation and substrate roughness due to chemical heterogeneities. Also, substrate hydrophobicity has been found to favour the stability of NBs [67]. These findings are in agreement with a series of recent experimental findings in the area of NBs, which will be discussed in more detail in subsequent sections.

\subsection{Simulation}

Molecular dynamics (MD) simulation has been used to study surface NBs, due to its flexibility in providing a molecular-level description in dense systems and the availability of reliable force-fields and highly parallelised free simulation software for both all-atom and coarse-grained (CG) models (see Fig. 33).

By means of MD simulation, Weijs et al. investigated the formation and stability mechanisms of bulk NBs in a binary mixture of simple Lennard-Jones (LJ) 
CG particles (see Fig. 3B) 69, 70]. The MD simulations were able to capture the formation and growth of NBs and estimate their final size allowing for a direct comparison of the results with theoretical predictions from nucleation theory. The authors concluded that NBs are stable as far as they are at a large enough distance between them so as to protect themselves from diffusion by a shielding effect, which is in agreement with simple diffusion calculations and experimental results based on noncoalescence observations for macroscopic bubbles [69]. Further work by Weijs et al. with MD simulation has underlined the importance of the hydrophobic nature of the substrate in order to favour the spontaneous formation of NBs [71. In this case, the observations from CG MD simulation align with the theoretical expectation of the dynamic equilibrium model, which suggests that NBs be stabilized by a nonequilibrium mechanism [70] requiring a balance of gas influx and outflux [51]. This CG model has provided LJ parameters that can capture the formation of NBs and can be used for further investigations based on NBs modelling [71. For example, Maheswari et al. have studied the stability and growth of NBs on chemically patterned substrates by using MD simulations based on the latter model [72]. The simulations revealed the importance of contact-line pinning and gas oversaturation for NBs stability. The latter studies have been recently extended to consider the case of two neighbouring surface NBs on a chemically heterogeneous substrate [73]. In this case, a diffusion equation-based stability analysis suggested that the NBs remain stable when the contact line is pinned with their radii of curvature being equal [73]. Li et al. have systematically studied the coalescence of two neighbouring NBs by means of CG MD simulation and analysed their results on the basis of different ratio of interactions between gas, liquid and substrate [74. When the contact line is not pinned, different coalescence scenarios are possible, such as direct merging through the existence of a film layer of gas molecules between the NBs. By means of MD simulations, Hong et al. [75] predicted that NBs of a certain radius $R$ are stable when the distance between bubbles is smaller than a length scale $L$ proportional to $R^{4 / 3}$, in this way providing an indication for the stable bubble concentration in solution. 
MD simulation has also demonstrated that surface NBs can be stabilised in superheated or gas oversaturated liquid due to the contact-line pinning that stems from surface heterogeneity [54]. Both the pinning and the oversaturation or superheating of the gas phase in liquid-vapour mixtures is a requirement for the stability of surface NBs according to this MD study, which is also currently a dominant assumption in the literature. Moreover, stable NBs are obtained at moderate oversaturation when the radius of curvature and the contact angle of the NBs decrease, whereas a liquid-vapour transition takes place at high oversaturation (supersaturation) 54. The simulations also predicted that both the gas-side radius of the curvature and the gas-side contact angle increase as the superheating/supersaturation levels increase. However, NBs become unstable at high levels of superheating/supersaturation [54].

The above assumptions have been supported by recent all-atom MD simulation, which has also provided details on the formation, dissolution and properties of surface NBs on an ideal HOPG substrate (see Fig. 3C) 68. NBs favourably formed on the substrate underlining the importance of the substrate and its hydrophobic nature that leads to the attraction of the air molecules. While NBs were formed through a nucleation process, they eventually dissolved, in this way providing evidence that their stability can be attributed to other mechanisms (e.g. contact-line pinning). Most importantly, this study has provided first-hand measurements for NBs density concluding that NBs consist of dense gaseous phases, which has been recently validated by experimental data [76]. Moreover, the gas-side contact angle of NBs (see Fig. 1) is smaller than that in nanodroplets (liquid-side) 68. This difference in contact angle between bubbles and droplets with size smaller than about $10 \mu \mathrm{m}$ has been recently studied by Zhang and Zhang by means of theory and MD simulation of a CG model [77. They found that this mismatch becomes more pronounced as the size of these nano-objects decreases. Moreover, the contact angle in the case of nanodroplets is size and model dependent [78. In addition to the contact-angle mismatch between nanodroplets and NBs, the all-atom MD simulation has found that the surface tension in NBs is smaller than the corresponding surface tension of a 
water-air interface under atmospheric conditions [68. A similar model has recently focused on the mechanical stability of surface NBs by Dockar et al. [79]. In this study, quasi-two-dimensional and three-dimensional nitrogen surface NBs were investigated and new cavitation threshold models were proposed and assessed. This study suggests that the discrepancies between experiments and the Blake threshold are due to the contact-line pinning, in this way providing further evidence for pinning as a plausible mechanism for stabilising surface NBs [79]. By means of all-atom MD simulation Chen et al. have recently investigated the stability of surface NBs on hydrophobic surfaces [80. They found that NBs were stable for longer than $160 \mathrm{~ns}$ and the stability depends on the gas adsorption, the solid-gas interaction energy, and the bulk gas concentration. Moreover, an increased hydrophobicity of the substrate alleviates the requirement of oversaturation conditions. The authors also suggest that the gas enrichment layer, the gas adsorption monolayer on the substrate, and the water hydrogen bonding near the interface could be the necessary conditions for the stability of NBs [80]. In contrast, they concluded that three-phase pinning sites are not necessarily required. All-atom simulation has also explored the midrange nanoscale hydrophobic interaction by NB nucleation between nanometre-sized hydrophobes [81. Koishi et al. 81 found that the NB formation exhibits hysteresis when the size of the hydrophobe is larger than $2 \mathrm{~nm}$. By means of potential-of-meanforce calculation, they were able to provide an estimation of the strength of the nanoscale hydrophobic interaction.

Given that the solvent exchange procedure (discussed in Section 4) has become a widely used protocol to produce surface NBs, MD simulation has attempted to study this process as well [82. In a recent study, Xiao et al. found that a solvent-solvent interface that traps gas molecules forms during the solvent exchange process $[82$. As this interface moves against the gas concentration gradient and towards the substrate, the local oversaturation of gas molecules initiate the nucleation of the NB on the substrate or in the bulk solution. Xiao et al. were able to draw a phase diagram of substrate wettability versus gas oversaturation, which indicates areas of favourable NBs formation 82. More- 
over, Xiao et al. have carried out further MD investigations to assess the effect of surfactants on the stability of surface NBs 83 . Their simulations suggest that the presence of surfactants may lead to the loss of stability of the NB as a result of either the adsorption of surfactant onto the substrate or the decrease of the vapour-liquid surface tension. This would lead to the depinning of the contact line, especially when the surfactants are water-insoluble. It is anticipated that this study may have further implications regarding the effect of other contaminants on the stability of gaseous phases at liquid-vapour interfaces.

A very recent work by Molinero et al., which highlights the predictive capabilities of CG MD simulation, has focused on the nucleation mechanisms as well as the formed stationary states of electrochemically generated NBs [84. The MD simulation is able to capture the molecular pathway of NB nucleation toward formation on nanoelectrodes and characterise the stationary states. In particular, the simulation indicates a classical mechanism for NB nucleation, where different nucleation regimes depend on the binding free energy per area of the NB to the electrode. This work predicts different states for the NB, such as micropancakes attached to the electrodes and homogeneously nucleated NBs close to the electrode without attachment. The authors conclude that NBs nucleate heterogeneously. Moreover, the stronger the driving force for the electrochemical reaction, the larger the size of the NB and the higher its contact angle with the electrode.

Finally, Many-body Dissipative Particle Dynamics (MDPD) simulation has explored the sliding dynamics of a NB on a surface indicating that the surface roughness and the bubble shape affect NB motion [85]. For small gas-side contact angles, NB mobility is higher even than the rising of a bulk NB, while below a contact-angle threshold-value, the mobility of a NB on a substrate with roughness can be higher than the mobility of a NB on a flat (smooth) substrate due to the superlyophobicity, which can reduce the friction resistance [85. 


\subsection{Experiment}

Before considering surface NBs, it is worthwhile to briefly discuss the stability of free (bulk) NBs [3]. In fact, much literature refers to bulk NBs, which can form spontaneously under different salt concentration and $\mathrm{pH}$ [86]. Bulk NBs have been generally found to be stable in the case of alkaline solutions, in contrast to what has been observed in solutions with high ionic strength [86]. Moreover, the zeta-potential has indicated that NBs have an electric double layer of negative charge, which is attributed to the adsorption of $\mathrm{OH}^{-}$at the gas-water interface. This electric double layer may prevent the aggregation of bubbles and reduce the surface tension, which, in turn, may decrease the internal pressure in the bubble. Moreover, the charged interface may lead to additional Maxwell pressure and further decrease of the surface tension, which may explain the reason that NBs are stable in aqueous solutions of water-soluble organic molecules of low molar mass [86]. Bulk NBs have been also studied in ultra-pure water solutions of $\mathrm{LiCl}$ and $\mathrm{NaCl}$ by means of Rayleigh/Brillouin scattering, highlighting a dependence of NB stability on salt concentration [87. Long lifetime and ageing effects in NBs were attributed to the electric charge, while their stability might be due to the coverage of their surface with negative ions 87.

In the case of surface NBs, Ishida et al. have carried out experiments on surface NBs by using tapping-mode AFM (TM-AFM) [10] (see Fig. 4A). In this case, a single wafer, hydrophobised with OTS, was immersed in water. The sensing mechanism of NB in TM-AFM is based on the repulsive force that produces a bump in the image, assuming that the AFM tip does not penetrate the bubble [9]. The height of the NBs was determined on the phase image and the force curves and the apparent contact angle (gas side) was estimated to be much smaller than the macroscopically expected contact angle. At that time, the small gas-side contact angle was believed to be one of the reasons for the stability of surface NBs [10], while theory currently suggests that surface NBs with gas-side contact angles smaller than $90^{\circ}$ can be stable 35. Further studies by TM-AFM have revealed close-packed NBs with irregular cross-sections and 
radii of curvature about $100 \mathrm{~nm}$ and height $20-30 \mathrm{~nm}[12,88$. NBs on a hydrophobic glass for a range of different $\mathrm{pH}$ conditions indicated that the increase of $\mathrm{pH}$ leads to smaller and more uniform NBs, which was attributed to the presence of the surface charge as in the case of bulk NBs [89. TM-AFM studies on atomically flat substrates have observed stable surface NBs for hours during experiments with the smallest NBs being around $10 \mathrm{~nm}$. Further experiments of surface NBs, which were formed for the first time by using the ethanol-water exchange method (nowadays a standard method to generate surface NBs discussed later in Section (4) on an OTS silicon hydrophobic substrate, have found results that are consistent with experiments of NBs on HOPG substrate [90]. By considering a wide range of solutes, such as multivalent salts, cationic, anionic, and nonionic surfactants, and solution $\mathrm{pH}$, Zhang et al. concluded that these factors influence very little the morphology of NBs [90]. In agreement with previous results, the gas-side contact angle of NBs was estimated to be smaller than the expected macroscopic angles on the same substrate suggesting that the Laplace pressure may be smaller, since a larger radius of curvature would imply a small pressure [90. Still, the predicted theoretical value of the pressure is smaller than the one obtained from experimental measurements [90. In the so-called force modulation mode of TM-AFM, which is based on the interaction between the cantilever tip and the bubble, the height and the adhesive force for the NB has been obtained 91. By using a viscoelastic model, the mechanical properties of NBs can be assessed by means of the stiffness and the damping coefficient. The modes were also set to study the effect of the surface tension on the attractive interaction force and the contact angle hysteresis during the tip-bubble interaction [91. Finally, Wang et al. 92 found that nanoindents are formed after immersing an ultrathin polystyrene film in water, and the size and location of the nanoindents are strongly correlated with that of NBs.

TM-AFM experiments have also been used to study the formation propensity of NBs and their distribution on an ultra-thin polystyrene (PS) film in deionized (DI) water and saline (sodium chloride) solution as a function of electrolyte, roughness, $\mathrm{pH}$, and substrate bias [93]. In this study, the saline environment 
favoured NBs of larger size in comparison with NBs in DI water. Increased roughness also led to larger bubbles. The study also confirmed previous results (for both bulk and surface NBs) that NBs are more stable in alkaline solutions than in acidic. Finally, the size of NBs was found to increase with positive bias 93. TM-AFM experiments have also considered the stability and coalescence of NBs with lateral size from $100 \mathrm{~nm}$ up to around $10 \mu \mathrm{m}$ and height from $10 \mathrm{~nm}$ to $300 \mathrm{~nm}$ on PS-water interface [94. It was found that the number of gas molecules increased by $112.5 \%$ after coalescence, which was attributed to the gas influx coming from the depinning of the contact line and the decrease of the inner pressure during coalescence [94. To this end, the coalescence was slower for larger bubbles. The results are consistent with the contact line pinning theory and experimental studies, which have estimated the lifetime to be about 6.9 hours in this case [94]. Hence, this study has provided support in favour of contact-line pinning, gas influx near the contact line and a thin 'contaminant film' around the gas-liquid interface, and even the electrostatic effect, in this way incorporating many elements of previous theories.

NBs on hydrophilic substrates immersed in water, such as self-assembled monolayers (SAMs) 95, have been observed by in situ AFM for the first time by Song et al. with gas-side contact angles larger than $94^{\circ}$ [96. In this case, the size of the NBs was found to depend on the composition of SAMs. By fitting the NB shape to a spherical cap, the height and the radii of the basis and the curvature were found in agreement with previous studies. In the case of hydrophilic SAMs, the macroscopic and microscopic contact angles also show agreement [96]. On the contrary, in the case of smooth hydrophilic dehydroxlylated silicon oxide wafer surfaces in water, TM-AFM experiments suggest that the formation of NBs is not possible [13. However, randomly distributed NBs were observed on methylated surfaces with controlled roughness, which, according to the authors, may indicate that the pinning of the contact line can stabilise the NB allowing for a small microscopic gas-side contact angle [13.

It should be noted that the invasive nature of AFM certainly affects the apparent shape of NBs. For this reason, Walczyk et al. have studied NBs on 
HOPG substrates in water by using both TM-AFM and PeakForce (PF) mode AFM 97]. In the case of PF-AFM, the force exerted on NBs was measured instead of the resonating cantilever with the apparent size and shape depending on the force. Even for forces as small as $73 \mathrm{pN}$, the true size of the NBs appeared to be smaller in the experiment with the error in the estimation increasing for larger NBs [97]. The height images obtained by PF-AFM indicated a decreasing apparent size of NBs with increasing scanning force, which has been also observed in TM-AFM experiments, but there are differences in the absolute values of the contact angles 97]. Results of PeakForce quantitative Nano-Mechanics (PF-QHM) measurements in the case of HOPG substrates, which were also confirmed with TM-AFM experiments, estimated the stiffness of the NB from 60 to $120 \mathrm{pN} / \mathrm{nm}$, with smaller NBs being stiffer 98. Moreover, results on the morphology between the PF-QHM method and TM-AFM were consistent 98]. The contact angle of NBs on HOPG in water has been estimated about $61 \pm 4^{\circ}$ (gas side) by TM-AFM provided that the cantilever is clean and the HOPG substrate smooth, otherwise angles (gas side) as low as $30^{\circ}$ can be observed due to the contamination that presumably introduced roughness on the HOPG substrate 99, 100]. Such contamination effects also affect the formation of NBs having provided further evidence for the contact-line pinning hypothesis for the stability of surface NBs [101]. In general, contamination issues are critical in the interpretation of NBs experiment. Berkelaar et al. have convincingly pointed out that NB-like objects can be induced by the use of disposable needles in which PDMS contaminated the water. Therefore, nano-objects that look and behave as NBs, in some cases they might simply be induced by contamination [101. The effects on the contact angle of NBs stemming from different contamination scenarios have been discussed in detail by An et al. [100.

Still, the above results should be put in perspective considering the effects of different cantilevers and AFM modes on the experimental results. In particular, it is widely accepted that experimental measurements of the shape and size of NBs depend on the cantilever properties. This has been concluded by a study where 15 different cantilevers were used [99]. Moreover, three different AFM 
modes, namely, tapping-mode, lift-mode, and force-volume-mode (FVM) were tested in the case of HOPG substrates in water [102]. It was found that the tip-bubble interaction strength depends on the position of the tip relative to the NB. The direction of the tip movement was also found to have hydrodynamic effects, and hence influences the deformation of the NB [102]. The hydrodynamic effects were more pronounced in the FVM, where the tip approaches the NB from above, In contrast, the hydrodynamic effects were less pronounced in the lift-mode, where the tip approaches the NB from the side. In addition to the tip shape and cleanness, the scanning model in AFM experiments is another factor that influences the morphology observations, especially when the tip movement is complex 102]. By combining TM- and FVM-AFM methods with hydrophilic and hydrophobic AFM tips, Walczyk et al. analysed the NB deformation and its interaction with respect to the tip position on the NB [102]. The results indicated that the tip-bubble interaction strength and the magnitude of the bubble deformation depend on the vertical and horizontal positions of the tip on the bubble in the case of hydrophobic tips [102]. Moreover, hydrophobic tips led to severe bubble deformation due to the permanent contact of the tip with the NB, which has also led to the stretch of the NB as the tip was moving away from the bubble centre. In contrast, a hydrophilic tip with no direct contact between the tip and the NB may reduce these effects, in this way allowing for reliable AFM images and NB dimensions measurements [102]. In this case, a thin liquid film formed between the tip and the bubble. The deformation of the NB in the vertical direction depended on both the vertical and the horizontal position of the tip, independently of the tip hydrophobicity. The deformation increased with decreased tip-sample separation distance, with a faster decrease in the periphery of the bubble. A flat profile for the NB was obtained, which suggested a Laplace pressure closer to values of the atmospheric pressure. Walczyk et al. suggested that these effects and the contact line pinning may explain the long lifetimes of NBs [103. In a recent study by Wang et al., the contact angle measurement of NBs on mica and molybdenite substrates was investigated and its dependence on the curvature radius of the AFM probe tip was discussed 
[104. The authors concluded that the true contact angle is not always obtuse and largely affected by the tip curvature it is rather closer to the microscopic value.

Despite all the efforts by means of AFM experiments that have provided important information on the morphology of NBs due to the very good spatial resolution, AFM has certain drawbacks, such as the intrusiveness and the inability to provide information on the chemical identity of the NBs as AFM is not able to distinguish between NBs and other objects. Another disadvantage is the time-consuming full-size images and the inability to capture any transient effects, such as the formation of NBs, and the requirement of a mechanically and chemically stable environment for the measurements. These are a few of the reasons that additional methods have been employed individually or in combination with AFM techniques to complement the study of surface NBs (see Fig. 4.

In one of these methods, Attenuated Total Reflection Fourier Transform Infrared (ATR-FTIR) and AFM can measure the gas transfer by using the infrared active $\mathrm{CO}_{2}$ gas [105]. The measurements show that the NB gas exchanges with the dissolved gas in the liquid phase. While $\mathrm{CO}_{2} \mathrm{NBs}$ eventually dissolve, they remain stable for hours and the dissolution rate depends on the initial size of the NBs. In this study, pinning of the contact line was underlined as the main factor for the stability of surface NBs [105]. In another study, the pinning of NBs was again highlighted as the main reason for NBs stability by using AFM, optical microscopy and a high-speed camera in the case of OTS glass at $37^{\circ} \mathrm{C}$ [106].

Nonintrusive interference-enhanced reflection microscopy has also been used to visualise individual NBs (see Fig. $4 \mathrm{~B}$ ), which has shown that their formation is not provoked by the intrusive nature of the AFM technique [32, contrary to some experimental assumptions [97. Moreover, the growth dynamics of NBs can be investigated observing stable NBs with gas-side contact angles up to $35^{\circ}$. This method combined with particle tracking techniques can describe the flow of liquid in the vicinity of the NBs [32. Furthermore, the total-internal-reflection- 
fluorescence excitation method (see Fig. $4 \mathrm{C}$ ) has been used to track the NB formation on a hydrophilic glass during water-ethanol exchange resulting in strongly contrasting images with high spatial resolution [33. The nucleation dynamics during the solvent exchange procedure was resolved and a Brownian motion was observed for tracer particles near the NBs [33].

In situ Transmission Electron Microscopy (TEM) has been used to investigate electrolytically generated $\mathrm{H}_{2} \mathrm{NBs}$, where the gas initially dissolved in the solution and then nucleated near Au electrodes [107. The growth dynamics of the NBs indicated a dependence on the substrate roughness, while dewetting (dissolution) appeared to be driven by a wetting instability [107. The growth dynamics of small bubbles were not affected by neighbouring NBs, contrary to what may happen for larger NBs [108. In the case of graphene liquid cells (water encapsulated by graphene membrane), NBs were investigated by in situ Ultra-High Vacuum Transmission Electron Microscopy (UHV-TEM, see Fig. 4D) indicating two distinct growth mechanisms for NBs, which depend on their relative size and the existence of a critical radius [109. In fact, the liquid cell electron microscopy is a new technique for in situ imaging and control of nanoscale phenomena to study nanoscale processes in liquids [110]. Results have shown that radiolysis is generally important and hydrogen and hydrated electrons can achieve equilibrium concentrations within seconds, while heating is typically insignificant [110]. A significant advantage of this method is the ability to image bubble nucleation, growth and migration. Based on a simplified reaction-diffusion model, Grogan et al. could predict the conditions for the formation of $\mathrm{H}_{2}$ bubbles [110].

The chemical identity, phase state, and density of NBs can be analysed by means of Infrared Spectroscopy. This method has been applied in the case of $\mathrm{CO}_{2}$ NBs [23]. The gas phase for different gas solubilities is in atmospheric pressure, which might explain the high stability of NBs [23]. Results coming from Attenuated Total Reflection Infrared Spectroscopy in the case of $\mathrm{CO}_{2}$ estimated the dimensions of bubbles in the range 5-80 nm, with bubbles being stable for 1-2 hours 24]. In this case, the pressure of the gas phase was estimated to be 
similar to the ambient pressure. Further AFM experiments, which calculated the pressure on the basis of the radius of curvature and the bubble dimensions agree with the results based on the IR spectrum. Moreover, the lower pressure of $\mathrm{CO}_{2}$ in atmosphere and its greater solubility in water in comparison with $\mathrm{N}_{2}$ and $\mathrm{O}_{2}$ have been indicated as the main factors for explaining the lower stability in the case of $\mathrm{CO}_{2} \mathrm{NBs}$ 24]. Smaller NBs were found to have shorter lifetimes, while average pressure and curvature of NB decreased with time. Finally, experiments of plasmon resonance provide evidence that NBs are in gas phase and their low gas-side contact angle suggested that an attractive force acts between the solid-air and liquid-air interfaces [24. Previous Surface Plasmon Resonance (SPR) experiments in the context of a film had confirmed a low refractive index at the interface with the average pressure being estimated about $1 \mathrm{~atm}$, which is generally consistent with the observed radius of curvature $(R \sim 4 \mu \mathrm{m})$ in NBs and possibly explains their long lifetime 23 .

Quartz Crystal Microbalance (QCM) can be used to investigate the kinetics of adsorption of $\mathrm{CO}_{2}$ molecules dissolved in water on a hydrophobised silica surface and results have been compared with those obtained by AFM experiments [13, 111. The results indicated an early adsorption of the gas ( $<20 \mathrm{~min})$ before this could be detected by TM-AFM. Hence, the sensitivity to detect low surface coverage of NBs was much greater in the case of QCM than in the case of AFM, in this way allowing for a more detailed study of the kinetic process of NBs formation [26. The process consisted of two different consecutive stages, namely a slow and a fast adsorption process. The slow process was associated with the diffusion of gas molecules from the interfacial region to the surface (Harvei nuclei). After a NB reached a critical size, the gas adsorption took place through diffusion from the interfacial regions towards the NB. Results from QCM experiments on bare and thiol-coated gold surface by using the solvent-exchange method showed that the formation of NBs takes place within less than a minute [26. In this context, QCM has also highlighted possible applications of NBs in efficient cleaning of solid-liquid interfaces to remove bovine albumin, which takes place in a 10-second treatment, in contrast to the treatment with SDS 
(sodium dodecyl sulfate) surfactants that requires about $20 \mathrm{~min}$ [112.

Finally, Small-Angle X-ray Scattering (SAXS) experiments have investigated the formation of NBs on hydrophobic SAMs surface in a binary ethanol/water titration [113. SAXS revealed an electron density depletion layer at the hydrophobic interface with changing air solubility in the immersing liquid due to the NB formation [113. Hence, NB formation was responsible for the so-called long-range hydrophobic force [113. Other methods to study NBs include the Fluorescence Lifetime Imaging Microscopy (FLIM, see Fig. 4 $4 \mathrm{E}$ ) combined with AFM to investigate the impact of surface treatment and modification on surface NB nucleation [31. Also, Neutron Reflectivity measurements have been used to measure the water density in the interface region [28, 114].

In summary, two different types of methods have been discussed here regarding the experimental characterisation of surface NBs. The first type of methods is based on AFM, which offers very high spatial resolution but low temporal resolution. Hence, dynamic properties and the temporal evolution of the NB formation are challenging. Moreover, AFM experiments do not distinguish between NBs and other nano-objects and are generally unable to provide information on the chemical identity of NBs. These are invasive methods in nature, which affect the shape of NBs and results depend on cantilevers (e.g. hydrophobic or hydrophilic) and AFM modes (e.g. tapping, Peak Force, etc.). In addition, AFM experiments also require stable chemical and mechanical environments. The second type of methods is mainly optical and non-intrusive. These methods offer lower spatial resolution than AFM, but can achieve high temporal resolution. They offer continuous monitoring of relevant processes, such as bubble nucleation and growth with the ability to describe the kinetics of such processes. Combined with particle tracking methods can describe the flow of liquid in the vicinity of NBs. Finally, these methods are able to provide analysis on the chemical identity, phase state and density of NBs. Non-intrusive methods with high spatial and temporal resolution may constitute an ideal tool for future research in the area of surface NBs. 


\section{Morphological characteristics}

The morphology of NBs is usually characterised by their size dimensions that determine the contact angle. Information about these properties is mainly obtained by AFM experiments and applying fitting procedures [97, 99. In general, a spherical-cap shape is implied to describe surface NBs with a circular three-phase boundary, height of a few tens of nanometres and lateral extension of up to several microns [115. As a result, the contact angle (measured from the gas side) is expected to be small [97, 99]. Although the three-phase contact line of NB is usually circular, irregular (noncircular) shapes have been observed 90. Below, we discuss results related to the morphological and mechanical properties of NBs.

In fact, results on the contact angle of NBs (Fig. 1) vary and different values have been reported in the literature. While early experiments have found values of about $20^{\circ}$ on HOPG substrates [111, 115, Borkent et al. have measured contact angles of about $60^{\circ}$ by means of AFM experiments [99, which are among the highest values reported in the literature. However, the contact angle is also expected to depend on NB's size when its radius is smaller than $R=20 \mathrm{~nm}$ 99, which is in agreement with previous AFM experiments [90. When the roughness of the substrate increases, the contact angle can obtain values around $30^{\circ}$. Still, contact angles (gas side) measured by PFT-AFM indicate smaller values (i.e. between $5^{\circ}$ and $35^{\circ}$ ), which has been comparable with a range $5^{\circ}-20^{\circ}$ measured more recently by TM-AFM [97. The difference between microscopic and macroscopic contact angles on the same substrate has been encountered by both experiment [90] and simulation [68, where differences have been partly attributed to the line tension [10, 13. In the case of nitrogen NBs with average diameters 10-100 nm on $\mathrm{Au}(111)$ substrates, it has been experimentally shown that the line tension can change the sign from negative to positive as the NB size decreases [65, which underlines the role of the line tension in the case of small NBs. This effect had been already confirmed in the very first experiments as well as in the case of more recent experimental studies [9, 11, 12, 14, 96- 
[99, 116]. This suggests a larger radius of curvature in the case of NBs and a commensurate decrease in the Laplace pressure [90, which eventually leads to the modification of Young's equation [117-119] in order to account for the influence of the line tension [120 122. In fact, Yang et al. proposed that the line tension might be responsible for the difference between nanoscopic and macroscopic contact angles [13, which has also been discussed in the context of nanodroplets 123 126]. Still, the experimental verification of the line tension contribution is a challenging task [13, 90]. By collecting data from about 200 NBs at room temperature, Zhang et al. suggested that surface NBs on HOPG substrates that are larger than $100 \mathrm{~nm}$ in height and $2 \mu \mathrm{m}$ in curvature radius are rather unstable [115]. On mica substrates, the radius of the basis was less than $300 \mathrm{~nm}$ for air NBs, while air and hydrogen NBs on HOPG were in the range 50-450 nm [115]. On heated substrates, NBs on HOPG have exhibited larger lateral sizes of about $8 \mu \mathrm{m}$ with all NBs having a similar aspect ratio independently of the substrate temperature, which corresponded to a gas-side contact angle of $3^{\circ}-24^{\circ}$ [127].

Results on a crystalline (111) Si wafer coated with a thick PS film (hydrophobic substrate) have indicated that the radius of curvature of the NBs were of the order of $300 \mathrm{~nm}$ in this case [14]. Moreover, a spherical-cap shape has been assumed for the NBs and the apparent pressure has been estimated as higher than the atmospheric pressure (about $4.5 \mathrm{~atm}$ ) [14. In similar studies by Tyrell et al., curvature radii of the order of $100 \mathrm{~nm}$ and height 20-30 nm for NBs have been reported [12.

In the case of OTS-coated substrates, the estimated gas-side nanoscopic contact angle of NBs was about $12 \pm 9^{\circ}$ and on HOPG $16 \pm 6^{\circ}$, while the corresponding contact angles for nanodroplets were $72 \pm 5^{\circ}$ for OTS and $108 \pm 11^{\circ}$ for HOPG [90. The formation of NBs was facilitated when the solvents (ethanol) and water were at $45^{\circ} \mathrm{C}$ than at room temperature 90]. Finally, the average height of the NBs was $26 \mathrm{~nm}$ and the lateral size about $591 \mathrm{~nm}$ [90].

The mechanical properties of NBs on HOPG can be mapped with high resolution by means of PF-QHM (PeakForce Quantitative Nano-Mechanics) AFM 
98. The stiffness of the NBs lies within 60 and $120 \mathrm{pN} / \mathrm{nm}$. This behaviour was size-dependent with bigger NBs being softer than the smaller ones [98. Measurements from TM-AFM suggest that the NBs be harder than the corresponding nanodroplets 66].

The density of nanobubbles has been estimated by means of all-atom MD simulation. The obtained value was $409 \mathrm{~kg} / \mathrm{m}^{3}$ [68, which is of the order of the density of liquids, in agreement with recent experiments [76]. Moreover, all-atom MD simulation has found that the surface tension in the case of NBs is about $50 \mathrm{mN} / \mathrm{m}$, a value smaller than the water-air interface tension at atmospheric conditions 68.

\section{Formation of nanobubbles}

Nanobubbles can form spontaneously by simple immersion of a solid substrate [13, 14, 62, 111, 128, 130, Although the oversaturation of dissolved gas is not a requirement for nucleation and formation of NBs, a certain gas concentration (100-110\% gas concentration) and temperature of the liquid (between $25^{\circ}$ and $45^{\circ}$ ) seems to favour the formation of NBs [63, while the range of temperature for NBs formation appears to only depend weakly on the type of gas [131.

Despite the possibility of spontaneous formation, the solvent exchange method is commonly used to form NBs (see Fig. 5A) [15, 21, 132, which has been applied in the case of substrates with different chemical and physical properties [27, 32, 33, 90, 111, 113, 133, 137. In the case of growing microbubbles on highly ordered hydrophobic microcavity arrays with the solvent exchange method, bubbles self-organise into symmetric patterns, whereas asymmetric patterns were observed in the case of larger distance between the microcavities [132]. NBs as small as $10 \mathrm{~nm}$ have been reported by using the solvent-exchange method. In this process, deionized water (DI water) is firstly injected into the liquid cell and then replaced with pure ethanol 9 . After waiting for several minutes, the ethanol is replaced with DI water and many NBs can be produced. The under- 
lying principle of this method is the gas separation during the mixing process of the two liquids, due to its different solubility [9. The advantage of this approach is that any other contamination is generally avoided. To this end, 1-propanol has been mostly used in the solvent-exchange process by showing the largest increase in range followed by ethanol and methanol [22]. According to recent MD simulations, the oversaturation of the gas either on the substrate or in the bulk solution, which depends on substrate hydrophobicity and the degree of local gas oversaturation, may be the factor that favours the formation of the NBs in the case of the solvent-exchange process [68, 82]. Although the solvent-exchange method has high repeatability and generally low or no contamination, AFM has imaged organic pollutants introduced by the alcohol, which may even obscure the evidence of the existence of NBs [138]. For this reason, the Temperature Difference Method has been used to address such shortcomings by avoiding the use of alcohol, as has been shown, for example, in the case of NBs on HOPG substrates [138. Here, low-temperature water is replaced by high-temperature water and NBs form during the mixing process (see Fig. 5B) [138, 139. Finally, similarly to the solvent-exchange method, the saline solution/water exchange method has produced NBs for different concentrations and valences of saline liquids (see Fig. 5C) [140.

Surface NBs can be also formed during photochemical or electrochemical processes. For example, photocatalytic reactions can produce surface NBs by generating hydrogen in methanol/water solution when UV light illuminates a $\mathrm{TiO}_{2}$ coated substrate [141]. The produced NBs existed during the photocatalytic reaction [141]. In the case of electrolysis of water to form NBs on HOPG substrates, the HOPG substrate acts as a negative (positive) electrode to produce hydrogen (oxygen) NBs with the coverage and size of NBs increasing with the voltage [142. In this approach, water with a small amount of sodium chloride allows for larger currents, but results are similar as in the case of pure water 142. The existence of electrochemically generated NBs by hydrogen gas on HOPG have been initially confirmed by Zhang et al. 143. It has been demonstrated that the formation and growth of NBs was controlled 
by the applied voltage or the reaction time. Moreover, the authors were able to observe the formation, growth, coalescence and eventual release of merged NBs from the HOPG substrate [143]. Electrochemical generation of individual $\mathrm{H}_{2}, \mathrm{~N}_{2}$, and $\mathrm{O}_{2} \mathrm{NBs}$ have been reported as well in the literature [144 151. In particular, Liu et al. has demonstrated by means of experimental and numeical methods that a single NB at a Pt nanodisk electrode is sustainable due to the $\mathrm{H}_{2}$ electrogeneration and better agreement between experiment and simulation is achieved when a recessed electrode geometry is assumed [151]. In the case of individual $\mathrm{O}_{2} \mathrm{NBs}$ generated by electrooxidation of hydrogen peroxide $\left(\mathrm{H}_{2} \mathrm{O}_{2}\right)$, a minimum concentration of $\mathrm{O}_{2}$ is required to observe NB nucleation at the Pt electrode surface, which is about 130 times larger than the equilibrium saturation concentration of $\mathrm{O}_{2}$ [150]. Moreover, NBs can be generated for both positive and negative potentials. In this work, Ren et al. were also able to generate alternatingly single $\mathrm{O}_{2}$ and $\mathrm{H}_{2} \mathrm{NBs}$ within the same experiment, in this way allowing for a direct comparison of critical concentrations for nucleation [150]. German et al. have provided measurements of the Laplace pressure of single NBs with radius between 7 and $200 \mathrm{~nm}$ showing a linear relationship between NB's Laplace pressure and its reciprocal radius, in agreement with the classical thermodynamic description [149. German et al. have also measured the lifetime of individual hydrogen and nitrogen NBs by using a fast-scan electrochemical technique [148. In particular, they found that the dissolution of NBs is partly limited by the translocation of molecules across the gas-water interface, where the interfacial gas transfer is estimated to be around $10^{-9} \mathrm{~mol} /(\mathrm{N} \cdot \mathrm{s})[148$. Chen et al. have studied the nucleation and stability of individual electrochemically generated $\mathrm{H}_{2}$ and $\mathrm{N}_{2} \mathrm{NBs}$ at platinum nanoelectrodes [146, 147. In the case of $\mathrm{H}_{2} \mathrm{NBs}$, similarly to $\mathrm{O}_{2} \mathrm{NBs}$ a large concentration of gas at the electrode favours nucleation of NBs [146]. Moreover, the addition of surfactants would decrease the nucleation barrier and an amount of surfactant will accumulate at the $\mathrm{H}_{2}$-solution interface hindering the transfer of $\mathrm{H}_{2}$ molecules to the solution. As a result, the residual current reduces when the concentration of surfactant increases [145, 146]. Moreover, Luo and White suggest a two-step mechanism 
for the nucleation and growth of NB [145]. In the case of nitrogen NBs [147], the nucleation and growth of a single NB was studied. It was also found that the size of a stable NB nucleus depends on the concentration of the gas at the electrode, which is two orders of magnitude larger than the saturation concentration at room temperature and atmospheric pressure [147]. Also, the residual current after $\mathrm{NB}$ formation was proportional to the $\mathrm{N}_{2} \mathrm{H}_{4}$ concentration and the nanoelectrode radius, which indicates that the dynamic equilibrium depends on the $\mathrm{N}_{2} \mathrm{H}_{4}$ electrooxidation at the three-phase contact line [147]. Spontaneous reaction between hydrogen and oxygen within bubbles of diameter smaller than $150 \mathrm{~nm}$ has been also reported in the literature, as a result of the high Laplace pressure and fast dynamics of the gas atoms within the bubble [152, 153]. In terms of applications, hydrogen NBs produced through electrochemical reactions can be further used as a template for the synthesis of metal hollow nanoparticles [44] or nanoporous thin films [48.

\section{Stability: Contact-line pinning}

The stability of surface NBs is indicated by their lifetime. A long lifetime may imply a duration from a few hours to many days, which is beyond the expectation of the immediate dissolution of surface NBs due to the theoretically predicted high Laplace pressure. To this end, the currently dominant explanation in the literature for the high stability of surface NBs is the combined effect of contact line pinning and gas oversaturation, which we will discuss here in more detail.

Initially, it was observed that the formation of NBs is sensitive to the surface topography, as has been shown in the case of NBs on an HOPG substrate [154. NBs were only formed at the upper side of the atomic stops (hydrophobic areas), while no NBs were observed on the more hydrophilic areas with the highest coordinating atoms (large number of neighbours) [154]. In this case, the welldefined topography of the HOPG substrate was ideal to assess the effect of substrate roughness [154]. Thus, it was concluded that the substrate roughness 
appeared to favour the formation of NBs, in contrast to smooth hydrophilic substrates, such as dehydroxylated silicon oxide wafer substrates [13]. These experiments [13, 154] constituted an early indication for the significance of the contact-line pinning in rough substrates for the stabilisation of surface NBs.

Despite these earlier observations, the pinning assumption was established by Zhang et al. 30, Weijs et al. [52, and Liu et al. 67]. By using AFM, Zhang et al. suggested that the three-phase boundary of NBs were pinned during their morphological evolution (see Fig. 6] [30. Moreover, the saturation levels of the dissolved gas affect the NB lifetime and pinning of the contact line slows down the kinetics of both the growth and shrinking processes. Based on these observations, a bespoke 1D Epstein-Plesset model of gas diffusion was proposed, which included the effect of pinning [30]. However, the origin of the boundary of the pinning was still not completely understood at the time. A subsequent theoretical model tried to explain NBs stability by assuming a limited gas diffusion, the cooperative effect of NB clusters and the pinned contact line, which lead to a slower dissolution rate [52, in agreement with recent theoretical arguments on the collective dissolution of microbubbles [155]. The model did not require fitting or uncontrolled assumptions to obtain the lifetimes of NBs and predictions were in agreement with the experimental findings [52]. On the contrary, molecular-level simulations have indicated that NBs have shorter lifetimes (i.e. $100 \mathrm{~ns})$ due to the limited diffusion stemming from the limited system sizes, in contrast to the long lifetimes of experimental NBs [52, 68. While contact-line pinning is important for the stability of single surface NBs, it also plays an important role in suppressing the Ostwald ripening process between neighbouring NBs [156], which could result in the growing of small NBs (large curvature) and the shrinking of large NBs (small curvature). This mechanism can explain the different radii of curvature of NBs occurring in the case of a population of neighbouring surface NBs.

The pinning force can be experimentally estimated by pulling NBs with an AFM tip and monitoring the mechanical response with Total Internal Reflection Fluorescence Microscopy [157. This force has been recently estimated of the 
order of $0.1 \mu \mathrm{N}$, which is the force required to unpin the NB from its substrate [157. The force estimation is limited by the stability of the neck pulled from the bubble and is enhanced by the hydrophobicity of the tip. In particular, the more hydrophobic the tip, the larger the pinning forces that can be measured [157. The measured pinning force is in agreement with previous theoretical work in the literature, such as estimates from a lattice density functional theory [53.

Evidence for the contact-line pinning also stems from MD simulation of a CG model, which has demonstrated that surface NBs can be stabilised in superheated or gas-oversaturated liquid due to the substrate heterogeneity [54]. In this case, the oversaturation refers to superheating for pure liquids and gas oversaturation or superheating in the case of gas-liquid mixtures, with both conditions having the same effect on NBs stability [54]. Still, pinning is currently believed to be the main reason for the stability of surface NBs, while hydrophobicity of the substrate and oversaturation seem to play secondary roles [158]. Hence, in the model by Tan et al. only the pinning of the contact line is considered and is strictly required for the stability of NBs [158. Still, hydrophobicity and oversaturation can enhance this stability, due to the hydrophobic attraction between the substrate and the gas [158, in agreement with recent MD simulations [68. In fact, experiments have shown that NBs can also exist in open systems and undersaturated environments [158].

Further work by numerical simulation has underlined the role of contact-line pinning in the stability of NBs. Liu and Zhang proposed a mechanism for the three-phase contact-line pinning to obtain stable NBs, which result from the intrinsic nanoscale physical roughness or chemical heterogeneity of the substrate 67. By using classical DFT, it has been shown that NBs are in thermodynamic metastable states [67], in agreement with MD simulation [68. This theoretical assumption is consistent with nucleation theory and can predict relationships between the contact angle and size of the NBs, as well as the chemical potential [67. The critical nucleus can be stabilised due to pinning and its size can be estimated [67, along the magnitude of the pinning force itself [53]. In the case 
of stable NBs, the contact angles are independent of the substrate chemistry as its effects are cancelled out by the pinning, in agreement with experimental observations [53. Moreover, a two-step process can explain the NB nucleation based on the crevice model, i.e. entrapped air pockets in surface cavities that grow by diffusion [159]. In the latter study by Wang et al., the authors have provided direct evidence of spontaneous NB formation as the substrate is im-

mersed in water. In this case, the size and shape of the nanostructures play a role. For example, non-circular nanopits lead to NBs with non-circular footprint, which shows that strong pinning forces at the three-phase contact line [159. The effect of substrate hydrophobicity, where NBs are formed by the ethanol-water exchange method has been considered in the study by Zou et al. by studying an dodecyltrichlorosilane (DTS), an OTS, and an HOPG substrate [160]. The authors found that increased hydrophobicity favours smaller contact angles (gas side), which is expected given the hydrophobic character of the gas phase. Moreover, nanoscopic and macroscopic contact angles match when the substrate hydrophobicity increases [160].

Finally, the disjoining pressure originating from the van der Waals interactions of the liquid and the gas with the substrate can affect the properties of NBs 161. In particular, it is believed that the disjoining pressure restricts the aspect ratio (lateral size/height) of the NB and a maximal aspect ratio exists [161. The influence of the disjoining pressure on the NB shape is minimal predicting a spherical-cap shape for individual NBs, in this way confirming early assumptions from AFM experiments [161].

\section{Perspectives}

The main assumption for the unexpected high stability of surface NBs has been the contact-line pinning caused by topological and chemical heterogeneities on the surfaces. Still, AFM cannot distinguish between the NBs and other objects and conclusions may be affected by this limitation. Non-intrusive methods with both high spatial and temporal resolutions would be ideal. Different sim- 
ulation methods can provide details on the mechanisms of NBs, such as MD, classical DFT, Monte Carlo, and others, which may also provide further information on various physical, chemical, mechanical, and thermodynamic properties of surface NBs. Examples of various properties and parameters of interest may be the distribution of charges, electric double layers, molecular structures, the presence of contaminants, surface-active additives, surface energy (wettability), and others. Moreover, there currently exists a limited selection of studied substrates in the literature, such as HOPG and graphene. It would probably be worthwhile to conduct experiments on different substrates in the future. Further experiments investigating the interaction of NBs with external fields, such as electric and magnetic fields, temperature, acoustic, and pressure waves could also be of interest for a number of applications in the research area of surface NBs.

\section{Acknowledgements}

This project has received funding from the European Union's Horizon 2020 research and innovation programme under the Marie Skłodowska-Curie grant agreement No. 778104. The work is also supported by the National Natural Science Foundation of China (Grant No. 51676137) and the Natural Science Foundation of Tianjin City (Grant No. 16JCYBJC41100). This research is supported in part by PLGrid Infrastructure.

\section{References}

\section{References}

[1] D. Lohse, X. Zhang, Surface nanobubbles and nanodroplets, Reviews of Modern Physics 87 (3) (2015) 981-1035. doi:10.1103/RevModPhys.87. 981.

[2] H. Peng, G. Birkett, A. Nguyen, Progress on the surface nanobubble story: What is in the bubble? why does it exist?, Advances in Colloid and Interface Science 222 (2015) 573-580. doi:10.1016/j.cis.2014.09.004. 
[3] M. Alheshibri, J. Qian, M. Jehannin, V. S. J. Craig, A history of nanobubbles, Langmuir 32 (43) (2016) 11086-11100. doi:10.1021/acs. langmuir.6b02489

[4] V. S. J. Craig, Very small bubbles at surfaces-the nanobubble puzzle, Soft Matter 7 (2011) 40-48. doi:10.1039/C0SM00558D

[5] J. L. Parker, P. M. Claesson, P. Attard, Bubbles, cavities, and the longranged attraction between hydrophobic surfaces., The Journal of Physical Chemistry 98 (34) (1994) 8468-8480. doi:10.1021/j100085a029.

[6] A. Carambassis, L. C. Jonker, P. Attard, M. W. Rutland, Forces measured between hydrophobic surfaces due to a submicroscopic bridging bubble, Physical Review Letters 80 (1998) 5357-5360. doi:10.1103/ PhysRevLett.80.5357.

[7] W. Gong, J. Stearnes, D. Fornasiero, R. A. Hayes, J. Ralston, The influence of dissolved gas on the interactions between surfaces of different hydrophobicity in aqueous media Part II. A spectroscopic study, Physical Chemistry Chemical Physics 1 (1999) 2799-2803. doi:10.1039/ A900977I.

[8] G. E. Yakubov, H.-J. Butt, O. I. Vinogradova, Interaction forces between hydrophobic surfaces. attractive jump as an indication of formation of stable submicrocavities, The Journal of Physical Chemistry B 104 (15) (2000) 3407-3410. doi:10.1021/jp000445+.

[9] S.-T. Lou, Z.-Q. Ouyang, Y. Zhang, X.-J. Li, J. Hu, M.-Q. Li, F.-J. Yang, Nanobubbles on solid surface imaged by atomic force microscopy, Journal of Vacuum Science \& Technology B: Microelectronics and Nanometer Structures Processing, Measurement, and Phenomena 18 (5) (2000) 25732575. doi:10.1116/1.1289925.

[10] N. Ishida, T. Inoue, M. Miyahara, K. Higashitani, Nano bubbles on a 
hydrophobic surface in water observed by tapping-mode atomic force microscopy, Langmuir 16 (16) (2000) 6377-6380. doi:10.1021/la000219r.

[11] S. Lou, J. Gao, X. Xiao, X. Li, G. Li, Y. Zhang, M. Li, J. Sun, X. Li, J. Hu, Studies of nanobubbles produced at liquid/solid interfaces, Materials Characterization 48 (2) (2002) 211 - 214. doi:10.1016/S1044-5803(02) 00241-3.

[12] J. W. G. Tyrrell, P. Attard, Images of nanobubbles on hydrophobic surfaces and their interactions, Physical Review Letters 87 (2001) 176104. doi:10.1103/PhysRevLett.87.176104.

[13] J. Yang, J. Duan, D. Fornasiero, J. Ralston, Very small bubble formation at the solidwater interface, The Journal of Physical Chemistry B 107 (25) (2003) 6139-6147. doi:10.1021/jp0224113.

[14] A. C. Simonsen, P. L. Hansen, B. Klösgen, Nanobubbles give evidence of incomplete wetting at a hydrophobic interface, Journal of Colloid and Interface Science 273 (2004) 291-299. doi:10.1016/j.jcis.2003.12. 035 .

[15] X. H. Zhang, X. D. Zhang, S. T. Lou, Z. X. Zhang, J. L. Sun, J. Hu, Degassing and temperature effects on the formation of nanobubbles at the mica/water interface, Langmuir 20 (9) (2004) 3813-3815. doi:10. 1021/la0364542.

[16] P. S. Epstein, M. S. Plesset, On the stability of gas bubbles in liquidgas solutions, The Journal of Chemical Physics 18 (11) (1950) 1505-1509. doi:10.1063/1.1747520.

[17] P. Ball, Chemical physics: How to keep dry in water, Nature 423 (6935) (2003) 25-26. doi:10.1038/423025a.

[18] X. H. Zhang, N. Maeda, J. Hu, Thermodynamic stability of interfacial gaseous states, The Journal of Physical Chemistry B 112 (44) (2008) 13671-13675, pMID: 18842008. doi:10.1021/jp807515f. 
[19] R. P. Berkelaar, H. J. W. Zandvliet, D. Lohse, Covering surface nanobubbles with a $\mathrm{NaCl}$ nanoblanket, Langmuir 29 (36) (2013) 11337-11343. doi:10.1021/la402503f.

[20] M. Holmberg, A. Khle, , K. A. Mørch, A. Boisen, Nanobubble trouble on gold surfaces, Langmuir 19 (25) (2003) 10510-10513. doi:10.1021/ 1a0352669.

[21] X. H. Zhang, Z. H. Wu, X. D. Zhang, G. Li, J. Hu, Nanobubbles at the interface of HOPG and ethanol solution, International Journal of Nanoscience 04 (03) (2005) 399-407. doi:10.1142/S0219581X05003152.

[22] M. A. Hampton, B. C. Donose, A. V. Nguyen, Effect of alcohol-water exchange and surface scanning on nanobubbles and the attraction between hydrophobic surfaces, Journal of Colloid and Interface Science 325 (1) (2008) 267 - 274. doi:10.1016/j.jcis.2008.05.044.

[23] X. H. Zhang, A. Khan, W. A. Ducker, A nanoscale gas state, Physical Review Letters 98 (2007) 136101. doi:10.1103/PhysRevLett.98.136101.

[24] X. H. Zhang, A. Quinn, W. A. Ducker, Nanobubbles at the interface between water and a hydrophobic solid, Langmuir 24 (9) (2008) 47564764. doi:10.1021/1a703475q.

[25] H. Seo, M. Yoo, S. Jeon, Influence of nanobubbles on the adsorption of nanoparticles, Langmuir 23 (4) (2007) 1623-1625. doi:10.1021/ $1 \mathrm{a} 062763 \mathrm{r}$

[26] X. H. Zhang, Quartz crystal microbalance study of the interfacial nanobubbles, Physical Chemistry Chemical Physics 10 (2008) 6842-6848. doi:10.1039/B810587A.

[27] J. Martinez, P. Stroeve, Transient behavior of the hydrophobic surface/water interface: from nanobubbles to organic layer, The Journal of Physical Chemistry B 111 (51) (2007) 14069-14072. doi:10.1021/ jp077110d. 
[28] R. Steitz, T. Gutberlet, T. Hauss, B. Klsgen, R. Krastev, S. Schemmel, A. C. Simonsen, G. H. Findenegg, Nanobubbles and their precursor layer at the interface of water against a hydrophobic substrate, Langmuir 19 (6) (2003) 2409-2418. doi:10.1021/la026731p.

[29] M. Switkes, J. W. Ruberti, Rapid cryofixation/freeze fracture for the study of nanobubbles at solid-liquid interfaces, Applied Physics Letters 84 (23) (2004) 4759-4761. doi:10.1063/1.1755837

[30] X. Zhang, D. Y. C. Chan, D. Wang, N. Maeda, Stability of interfacial nanobubbles, Langmuir 29 (4) (2013) 1017-1023. doi:10.1021/ 1 a303837c

[31] N. Hain, D. Wesner, S. I. Druzhinin, H. Schönherr, Surface nanobubbles studied by time-resolved fluorescence microscopy methods combined with AFM: the impact of surface treatment on nanobubble nucleation, Langmuir 32 (43) (2016) 11155-11163. doi:10.1021/acs .langmuir.6b01662

[32] S. Karpitschka, E. Dietrich, J. R. T. Seddon, H. J. W. Zandvliet, D. Lohse, H. Riegler, Nonintrusive optical visualization of surface nanobubbles, Physical Review Letters 109 (2012) 066102. doi:10.1103/PhysRevLett. 109.066102

[33] C. U. Chan, C.-D. Ohl, Total-internal-reflection-fluorescence microscopy for the study of nanobubble dynamics, Physical Review Letters 109 (2012) 174501. doi:10.1103/PhysRevLett.109.174501.

[34] C. U. Chan, L. Chen, M. Arora, C. D. Ohl, Collapse of surface nanobubbles, Physical Review Letters 114 (11) (2015) 1-5. doi:10.1103/ PhysRevLett.114.114505

[35] C. U. Chan, M. Arora, C. D. Ohl, Coalescence, growth, and stability of surface-attached nanobubbles, Langmuir 31 (25) (2015) 7041-7046. doi: 10.1021/acs.langmuir.5b01599 
[36] M. Hampton, A. Nguyen, Nanobubbles and the nanobubble bridging capillary force, Advances in Colloid and Interface Science 154 (1) (2010) 30 - 55. doi:10.1016/j.cis. 2010.01 .006

[37] D. J. Mastropietro, W. A. Ducker, Forces between hydrophobic solids in concentrated aqueous salt solution, Physical Review Letters 108 (2012) 106101. doi:10.1103/PhysRevLett.108.106101.

[38] N. Mishchuk, J. Ralston, D. Fornasiero, Influence of dissolved gas on van der waals forces between bubbles and particles, The Journal of Physical Chemistry A 106 (4) (2002) 689-696. doi:10.1021/jp0118221.

[39] S. Calgaroto, K. Wilberg, J. Rubio, On the nanobubbles interfacial properties and future applications in flotation, Minerals Engineering 60 (2014) 33 - 40. doi:10.1016/j.mineng.2014.02.002.

[40] D. Lohse, Bubble puzzles: From fundamentals to applications, Physical Review Fluids 3 (2018) 110504. doi:10.1103/PhysRevFluids.3.110504

[41] I. Kumagai, Y. Takahashi, Y. Murai, Power-saving device for air bubble generation using a hydrofoil to reduce ship drag: Theory experiments, and application to ships, Ocean Engineering 95 (2015) 183-194. doi: $10.1016 /$ j.oceaneng. 2014.11.019

[42] L. Chen, D. Rende, L. S. Schadler, R. Ozisik, Polymer nanocomposite foams, Journal of Materials Chemistry A 1 (2013) 3837-3850. doi:10. 1039/C2TA00086E

[43] S. Liu, J. Duvigneau, G. J. Vancso, Nanocellular polymer foams as promising high performance thermal insulation materials, European Polymer Journal 65 (2015) 33 - 45, 50 Years of European Polymer Journal. doi:10.1016/j.eurpolymj.2015.01.039

[44] C. Huang, J. Jiang, M. Lu, L. Sun, E. I. Meletis, Y. Hao, Capturing electrochemically evolved nanobubbles by electroless deposition. a facile 
route to the synthesis of hollow nanoparticles, Nano Letters 9 (12) (2009)

4297-4301. doi:10.1021/n1902529y.

[45] Z. Hammadi, R. Morin, J. Olives, Field nano-localization of gas bubble production from water electrolysis, Applied Physics Letters 103 (22) (2013) 223106. doi:10.1063/1.4836095

[46] E. Lukianova-Hleb, Y. Hu, L. Latterini, L. Tarpani, S. Lee, R. A. Drezek, J. H. Hafner, D. O. Lapotko, Plasmonic nanobubbles as transient vapor nanobubbles generated around plasmonic nanoparticles, ACS Nano 4 (4) (2010) 2109-2123. doi:10.1021/nn1000222.

[47] J. Zou, H. Zhang, Z. Guo, Y. Liu, J. Wei, Y. Huang, X. Zhang, Surface nanobubbles nucleate liquid boiling, Langmuir 34 (46) (2018) 1409614101. doi:10.1021/acs.langmuir.8b03290.

[48] F. Hui, B. Li, P. He, J. Hu, Y. Fang, Electrochemical fabrication of nanoporous polypyrrole film on HOPG using nanobubbles as templates, Electrochemistry Communications 11 (3) (2009) 639-642. doi:10.1016/ j.elecom.2008.12.051.

[49] S. Ljunggren, J. C. Eriksson, The lifetime of a colloid-sized gas bubble in water and the cause of the hydrophobic attraction, Colloids and Surfaces A: Physicochemical and Engineering Aspects 129 (1997) 151 - 155, prof. B. W. Ninham. doi:10.1016/S0927-7757(97)00033-2.

[50] W. A. Ducker, Contact angle and stability of interfacial nanobubbles, Langmuir 25 (16) (2009) 8907-8910. doi:10.1021/la902011v.

[51] M. P. Brenner, D. Lohse, Dynamic equilibrium mechanism for surface nanobubble stabilization, Physical Review Letters 101 (2008) 214505. doi:10.1103/PhysRevLett.101.214505

[52] J. H. Weijs, D. Lohse, Why surface nanobubbles live for hours, Physical Review Letters 110 (2013) 054501. doi:10.1103/PhysRevLett.110. 054501 . 
[53] Y. Liu, J. Wang, X. Zhang, W. Wang, Contact line pinning and the relationship between nanobubbles and substrates, The Journal of Chemical Physics 140 (5) (2014) 054705. doi:10.1063/1.4863448.

[54] Y. Liu, X. Zhang, A unified mechanism for the stability of surface nanobubbles: Contact line pinning and supersaturation, The Journal of Chemical Physics 141 (13) (2014) 134702. doi:10.1063/1.4896937

[55] D. Lohse, X. Zhang, Pinning and gas oversaturation imply stable single surface nanobubbles, Physical Review E 91 (2015) 031003. doi:10.1103/ PhysRevE.91.031003.

[56] B. H. Tan, H. An, C.-D. Ohl, Stability, dynamics, and tolerance to undersaturation of surface nanobubbles, Physical Review Letters 122 (2019) 134502. doi:10.1103/PhysRevLett.122.134502.

[57] X. Zhu, R. Verzicco, X. Zhang, D. Lohse, Diffusive interaction of multiple surface nanobubbles: shrinkage, growth, and coarsening, Soft Matter 14 (2018) 2006-2014. doi:10.1039/c7sm02523h.

[58] S. Das, J. H. Snoeijer, D. Lohse, Effect of impurities in description of surface nanobubbles, Physical Review E 82 (2010) 056310. doi:10.1103/ PhysRevE.82.056310.

[59] S. Das, Effect of impurities in the description of surface nanobubbles: Role of nonidealities in the surface layer, Physical Review E 83 (2011) 066315. doi:10.1103/PhysRevE.83.066315.

[60] X. Zhang, M. H. Uddin, H. Yang, G. Toikka, W. Ducker, N. Maeda, Effects of surfactants on the formation and the stability of interfacial nanobubbles, Langmuir 28 (28) (2012) 10471-10477. doi:10.1021/la301851g.

[61] S. M. Dammer, D. Lohse, Gas enrichment at liquid-wall interfaces, Physical Review Letters 96 (2006) 206101. doi:10.1103/PhysRevLett.96. 206101 . 
[62] J. R. T. Seddon, H. J. W. Zandvliet, D. Lohse, Knudsen gas provides nanobubble stability, Physical Review Letters 107 (2011) 116101. doi: 10.1103/PhysRevLett.107.116101.

[63] J. R. T. Seddon, E. S. Kooij, B. Poelsema, H. J. W. Zandvliet, D. Lohse, Surface bubble nucleation stability, Physical Review Letters 106 (2011) 056101. doi:10.1103/PhysRevLett.106.056101.

[64] N. D. Petsev, M. S. Shell, L. G. Leal, Dynamic equilibrium explanation for nanobubbles' unusual temperature and saturation dependence, Physical Review E 88 (2013) 010402. doi:10.1103/PhysRevE.88.010402.

[65] N. Kameda, S. Nakabayashi, Size-induced sign inversion of line tension in nanobubbles at a solid/liquid interface, Chemical Physics Letters 461 (1) (2008) 122 - 126. doi:10.1016/j.cplett.2008.07.012.

[66] N. Kameda, N. Sogoshi, S. Nakabayashi, Nitrogen nanobubbles and butane nanodroplets at $\mathrm{Si}(100)$, Surface Science 602 (8) (2008) 1579 - 1584. doi:10.1016/j.susc.2008.02.023

[67] Y. Liu, X. Zhang, Nanobubble stability induced by contact line pinning, The Journal of Chemical Physics 138 (1) (2013) 014706. doi:10.1063/ 1.4773249 .

[68] Z. Che, P. E. Theodorakis, Formation, dissolution and properties of surface nanobubbles, Journal of colloid and interface science 487 (2017) 123129. doi:10.1016/j.jcis.2016.10.027.

[69] J. H. Weijs, J. R. T. Seddon, D. Lohse, Diffusive shielding stabilizes bulk nanobubble clusters, ChemPhysChem 13 (8) (2012) 2197-2204. doi:10. 1002/cphc. 201100807 .

[70] J. H. Weijs, J. H. Snoeijer, D. Lohse, Formation of surface nanobubbles and the universality of their contact angles: A molecular dynamics approach, Physical Review Letters 108 (2012) 104501. doi:10.1103/ PhysRevLett.108.104501. 
[71] J. H. Weijs, J. H. Snoeijer, D. Lohse, Surface nanobubbles: Formation and universality of the contact angle, Physical Review Letters 108 (2012) 104501. doi:10.1103/PhysRevLett.108.104501.

[72] S. Maheshwari, M. Van Der Hoef, X. Zhang, D. Lohse, Stability of surface nanobubbles: A molecular dynamics study, Langmuir 32 (43) (2016) 11116-11122. doi:10.1021/acs.langmuir.6b00963.

[73] S. Maheshwari, M. van der Hoef, J. Rodríguez Rodrguez, D. Lohse, Leakiness of pinned neighboring surface nanobubbles induced by strong gassurface interaction, ACS Nano 12 (3) (2018) 2603-2609. doi:10.1021/ acsnano.7b08614.

[74] C. Li, S.-P. Wang, A.-M. Zhang, Y. Liu, Dynamic behavior of two neighboring nanobubbles induced by various gas-liquid-solid interactions, Physical Review Fluids 3 (2018) 123604. doi:10.1103/PhysRevFluids.3. 123604

[75] S.-N. Hong, S.-H. Choe, U.-G. Jong, M.-S. Pak, C.-J. Yu, The maximum interbubble distance in relation to the radius of spherical stable nanobubble in liquid water: A molecular dynamics study, Fluid Phase Equilibria 487 (2019) 45-51. doi:10.1016/j.fluid.2019.01.014

[76] S. Wang, L. Zhou, X. Wang, C. Wang, Y. Dong, Y. Zhang, Y. Gao, L. Zhang, J. Hu, Force spectroscopy revealed a high-gas-density state near the graphite substrate inside surface nanobubbles, Langmuirdoi: 10.1021/acs. langmuir.8b03383

[77] H. Zhang, X. Zhang, Size dependence of bubble wetting on surfaces: breakdown of contact angle match between small sized bubbles and droplets, Nanoscale 11 (2019) 2823-2828. doi:10.1039/c8nr08929a.

[78] P. E. Theodorakis, E. A. Müller, R. V. Craster, O. K. Matar, Modelling the superspreading of surfactant-laden droplets with computer simulation, Soft Matter 11 (2015) 9254-9261. doi:10.1039/c5sm02090E. 
[79] D. Dockar, M. K. Borg, J. M. Reese, Mechanical stability of surface nanobubbles, Langmuirdoi:10.1021/acs. langmuir.8b02887.

[80] Y.-X. Chen, Y.-L. Chen, T.-H. Yen, Investigating interfacial effects on surface nanobubbles without pinning using molecular dynamics simulation, Langmuir 34 (2018) 15360-15369. doi:10.1021/acs.langmuir.8b03016

[81] T. Koishi, S. Yoo, K. Yasuoka, X. C. Zeng, T. Narumi, R. Susukita, A. Kawai, H. Furusawa, A. Suenaga, N. Okimoto, N. Futatsugi, T. Ebisuzaki, Nanoscale hydrophobic interaction and nanobubble nucleation, Physical Review Letters 93 (2004) 185701. doi:10.1103/ PhysRevLett.93.185701.

[82] Q. Xiao, Y. Liu, Z. Guo, Z. Liu, D. Lohse, X. Zhang, Solvent exchange leading to nanobubble nucleation: A molecular dynamics study, Langmuir 33 (32) (2017) 8090-8096. doi:10.1021/acs.langmuir.7b01231.

[83] Q. Xiao, Y. Liu, Z. Guo, Z. Liu, X. Zhang, How nanobubbles lose stability: Effects of surfactants, Applied Physics Letters 111 (13) (2017) 131601. doi:10.1063/1.5000831.

[84] Y. A. Perez Sirkin, E. D. Gadea, D. A. Scherlis, V. Molinero, Mechanisms of nucleation and stationary states of electrochemically generated nanobubbles, Journal of American Chemical Societydoi:10.1021/jacs. $9 \mathrm{~b} 04479$.

[85] C. J. Wu, K. C. Chu, Y. J. Sheng, H. K. Tsao, Sliding dynamic behavior of a nanobubble on a surface, Journal of Physical Chemistry C 121 (33) (2017) 17932-17940. doi:10.1021/acs.jpcc.7b04924.

[86] F. Jin, J. Li, X. Ye, C. Wu, Effects of $\mathrm{pH}$ and ionic strength on the stability of nanobubbles in aqueous solutions of $\alpha$-Cyclodextrin, The Journal of Physical Chemistry B 111 (40) (2007) 11745-11749. doi: 10.1021/jp074260f 
[87] E. Duval, S. Adichtchev, S. Sirotkin, A. Mermet, Long-lived submicrometric bubbles in very diluted alkali halide water solutions, Physical Chemistry Chemical Physics 14 (2012) 4125-4132. doi:10.1039/C2CP22858K.

[88] P. Attard, M. P. Moody, J. W. Tyrrell, Nanobubbles: the big picture, Physica A: Statistical Mechanics and its Applications 314 (1) (2002) 696 - 705, horizons in Complex Systems. doi:10.1016/S0378-4371(02) 01191-3.

[89] J. W. G. Tyrrell, P. Attard, Atomic force microscope images of nanobubbles on a hydrophobic surface and corresponding force-separation data, Langmuir 18 (1) (2002) 160-167. doi:10.1021/la0111957.

[90] X. H. Zhang, N. Maeda, V. S. J. Craig, Physical properties of nanobubbles on hydrophobic surfaces in water and aqueous solutions, Langmuir 22 (11) (2006) 5025-5035. doi:10.1021/la0601814.

[91] B. Bhushan, Y. Wang, A. Maali, Coalescence and movement of nanobubbles studied with tapping mode AFM and tip-bubble interaction analysis, Journal of Physics: Condensed Matter 20 (48) (2008) 485004. doi:10.1088/0953-8984/20/48/485004.

[92] Y. Wang, B. Bhushan, X. Zhao, Nanoindents produced by nanobubbles on ultrathin polystyrene films in water, Nanotechnology 20 (4) (2009) 045301. doi:10.1088/0957-4484/20/4/045301.

[93] M. Mazumder, B. Bhushan, Propensity and geometrical distribution of surface nanobubbles: effect of electrolyte, roughness, ph, and substrate bias, Soft Matter 7 (2011) 9184-9196. doi:10.1039/C1SM05560G

[94] D. Li, D. Jing, Y. Pan, W. Wang, X. Zhao, Coalescence and stability analysis of surface nanobubbles on the polystyrene/water interface, Langmuir 30 (21) (2014) 6079-6088. doi:10.1021/la501262a 
[95] B. Song, Y. Zhou, H. Schönherr, Optimized model surfaces for advanced atomic force microscopy studies of surface nanobubbles, Langmuir 32 (43) (2016) 11179-11187. doi:10.1021/acs. langmuir.6b01776.

[96] B. Song, W. Walczyk, H. Schönherr, Contact angles of surface nanobubbles on mixed self-assembled monolayers with systematically varied macroscopic wettability by atomic force microscopy, Langmuir 27 (13) (2011) 8223-8232. doi:10.1021/la2014896.

[97] W. Walczyk, P. M. Schn, H. Schönherr, The effect of peakforce tapping mode AFM imaging on the apparent shape of surface nanobubbles, Journal of Physics: Condensed Matter 25 (18) (2013) 184005. doi:10.1088/0953-8984/25/18/184005.

[98] B. Zhao, Y. Song, S. Wang, B. Dai, L. Zhang, Y. Dong, J. Lu, J. Hu, Mechanical mapping of nanobubbles by peakforce atomic force microscopy, Soft Matter 9 (2013) 8837-8843. doi:10.1039/C3SM50942G.

[99] B. M. Borkent, S. de Beer, F. Mugele, D. Lohse, On the shape of surface nanobubbles, Langmuir 26 (1) (2010) 260-268. doi:10.1021/la902121x

[100] H. An, G. Liu, V. Craig, Wetting of nanophases: Nanobubbles, nanodroplets and micropancakes on hydrophobic surfaces, Advances in Colloid and Interface Science 222 (2015) 9-17. doi:10.1016/j.cis.2014. 07.008

[101] R. P. Berkelaar, E. Dietrich, G. A. M. Kip, E. S. Kooij, H. J. W. Zandvliet, D. Lohse, Exposing nanobubble-like objects to a degassed environment, Soft Matter 10 (2014) 4947-4955. doi:10.1039/C4SM00316K.

[102] W. Walczyk, N. Hain, H. Schonherr, Hydrodynamic effects of the tip movement on surface nanobubbles: a combined tapping mode, lift mode and force volume mode AFM study, Soft Matter 10 (2014) 5945-5954. doi:10.1039/C4SM01024H 
[103] W. Walczyk, H. Schönherr, Dimensions and the profile of surface nanobubbles: Tip-nanobubble interactions and nanobubble deformation in atomic force microscopy, Langmuir 30 (40) (2014) 11955-11965. doi:10.1021/ $1 \mathrm{a} 502918 \mathrm{u}$

[104] Y. Wang, X. Luo, W. Qin, F. Jiao, New insights into the contact angle and formation process of nanobubbles based on line tension and pinning, Applied Surface Science 481 (2019) 1585-1594. doi:10.1016/j .apsusc. 2019.01.292.

[105] S. R. German, X. Wu, H. An, V. S. J. Craig, T. L. Mega, X. Zhang, Interfacial nanobubbles are leaky: Permeability of the gas/water interface, ACS Nano 8 (6) (2014) 6193-6201. doi:10.1021/nn5016049.

[106] X. Zhang, H. Lhuissier, C. Sun, D. Lohse, Surface nanobubbles nucleate microdroplets, Physical Review Letters 112 (2014) 144503. doi:10.1103/ PhysRevLett.112.144503.

[107] Y. Liu, S. J. Dillon, In situ observation of electrolytic H2 evolution adjacent to gold cathodes, Chemical Communications 50 (2014) 1761-1763. doi:10.1039/C3CC46737F

[108] T.-W. Huang, S.-Y. Liu, Y.-J. Chuang, H.-Y. Hsieh, C.-Y. Tsai, W.-J. Wu, C.-T. Tsai, U. Mirsaidov, P. Matsudaira, C.-S. Chang, F.-G. Tseng, F.-R. Chen, Dynamics of hydrogen nanobubbles in KLH protein solution studied with in situ wet-TEM, Soft Matter 9 (2013) 8856-8861. doi: 10.1039/C3SM50906K.

[109] D. Shin, J. B. Park, Y.-J. Kim, S. J. Kim, J. H. Kang, B. Lee, S.-P. Cho, B. H. Hong, K. S. Novoselov, Growth dynamics and gas transport mechanism of nanobubbles in graphene liquid cells, Nature Communications 6 (2015) 6068. doi:10.1038/ncomms7068.

[110] J. M. Grogan, N. M. Schneider, F. M. Ross, H. H. Bau, Bubble and 
pattern formation in liquid induced by an electron beam, Nano Letters 14 (1) (2014) 359-364. doi:10.1021/nl404169a.

[111] J. Yang, J. Duan, D. Fornasiero, J. Ralston, Kinetics of CO2 nanobubble formation at the solid/water interface, Physical Chemistry Chemical Physics 9 (2007) 6327-6332. doi:10.1039/B709624K.

[112] G. Liu, Z. Wu, V. S. J. Craig, Cleaning of protein-coated surfaces using nanobubbles: An investigation using a quartz crystal microbalance, The Journal of Physical Chemistry C 112 (43) (2008) 16748-16753. doi:10. 1021/jp805143c.

[113] L. A. Palmer, D. Cookson, R. N. Lamb, The relationship between nanobubbles and the hydrophobic force, Langmuir 27 (1) (2011) 144-147. doi:10.1021/la1029678.

[114] D. Schwendel, T. Hayashi, R. Dahint, A. Pertsin, M. Grunze, R. Steitz, F. Schreiber, Interaction of water with self-assembled monolayers: neutron reflectivity measurements of the water density in the interface region, Langmuir 19 (6) (2003) 2284-2293. doi:10.1021/la026716k.

[115] L. Zhang, X. Zhang, Y. Zhang, J. Hu, H. Fang, The length scales for stable gas nanobubbles at liquid/solid surfaces, Soft Matter 6 (2010) 4515-4519. doi:10.1039/C0SM00243G

[116] X. Zhang, J. Ren, H. Yang, Y. He, J. Tan, G. G. Qiao, From transient nanodroplets to permanent nanolenses, Soft Matter 8 (2012) 4314-4317. doi:10.1039/C2SM07267J

[117] L. Boruvka, A. W. Neumann, Generalization of the classical theory of capillarity, The Journal of Chemical Physics 66 (12) (1977) 5464-5476. doi:10.1063/1.433866.

[118] P. G. de Gennes, Wetting: statics and dynamics, Reviews of Modern Physics 57 (1985) 827-863. doi:10.1103/RevModPhys.57.827. 
[119] P. G. de Gennes, On fluid/wall slippage, Langmuir 18 (9) (2002) 34133414. doi:10.1021/la0116342.

[120] A. Amirfazli, A. W. Neumann, Status of the three-phase line tension, Advances in Colloid and Interface Science 110 (3) (2004) 121-141. doi: $10.1016 /$ j.cis. 2004.05.001.

[121] T. Pompe, S. Herminghaus, Three-phase contact line energetics from nanoscale liquid surface topographies, Physical Review Letters 85 (2000) 1930-1933. doi:10.1103/PhysRevLett.85.1930

[122] T. Pompe, A. fery, S. Herminghaus, Measurement of contact line tension by analysis of the three-phase boundary with nanometer resolution, Journal of Adhesion Science and Technology 13 (1999) 1155-1164. doi:10.1163/156856199X00848.

[123] J. Zhang, P. Wang, M. Borg, J. M. Reese, D. Wen, A critical assessment of the line tension determined by the modified youngs equation, Physics of Fluids 30 (8) (2018) 082003. doi:10.1063/1.5040574.

[124] J.-C. Fernandez-Toledano, T. Blake, P. Lambert, J. De Coninck, On the cohesion of fluids and their adhesion to solids: Young's equation at the atomic scale, Advances in Colloid and Interface Science 245 (2017) 102107. doi:10.1016/j.cis.2017.03.006.

[125] R. Good, M. Koo, The effect of drop size on contact angle, Journal of Colloid Interface Science 71 (2) (1979) 283-292. doi:10.1016/ 0021-9797(79)90239-X.

[126] J. H. Weijs, A. Marchand, B. Andreotti, D. Lohse, J. H. Snoeijer, Origin of line tension for a lennard-jones nanodroplet, Physics of Fluids 23 (2) (2011) 022001. doi:10.1063/1.3546008.

[127] C. Xu, S. Peng, G. G. Qiao, V. Gutowski, D. Lohse, X. Zhang, Nanobubble formation on a warmer substrate, Soft Matter 10 (2014) 7857-7864. doi: 10.1039/C4SM01025F. 
[128] B. M. Borkent, S. M. Dammer, H. Schönherr, G. J. Vancso, D. Lohse, Superstability of surface nanobubbles, Physical Review Letters 98 (2007) 204502. doi:10.1103/PhysRevLett.98.204502.

[129] R. P. Berkelaar, J. R. T. Seddon, H. J. W. Zandvliet, D. Lohse, Temperature dependence of surface nanobubbles, ChemPhysChem 13 (8) (2012) 2213-2217. doi:10.1002/cphc.201100808

[130] A. Agrawal, J. Park, D. Y. Ryu, P. T. Hammond, T. P. Russell, G. H. McKinley, Controlling the location and spatial extent of nanobubbles using hydrophobically nanopatterned surfaces, Nano Letters 5 (9) (2005) 1751-1756. doi:10.1021/n10511030.

[131] M. A. J. van Limbeek, J. R. T. Seddon, Surface nanobubbles as a function of gas type, Langmuir 27 (14) (2011) 8694-8699. doi:10.1021/ la2005387

[132] S. Peng, T. L. Mega, X. Zhang, Collective effects in microbubble growth by solvent exchange, Langmuir 32 (43) (2016) 11265-11272. doi:10.1021/ acs.langmuir.6b02066.

[133] S. Yang, S. M. Dammer, N. Bremond, H. J. W. Zandvliet, E. S. Kooij, D. Lohse, Characterization of nanobubbles on hydrophobic surfaces in water, Langmuir 23 (13) (2007) 7072-7077. doi:10.1021/1a070004i.

[134] N. Ishida, Y. Kusaka, H. Ushijima, Hydrophobic attraction between silanated silica surfaces in the absence of bridging bubbles, Langmuir 28 (39) (2012) 13952-13959. doi:10.1021/la303037d.

[135] V. Belova, M. Krasowska, D. Wang, J. Ralston, D. G. Shchukin, H. Mohwald, Influence of adsorbed gas at liquid/solid interfaces on heterogeneous cavitation, Chemical Science 4 (2013) 248-256. doi:10.1039/ C2SC21321D 
[136] X. Zhang, N. Maeda, Interfacial gaseous states on crystalline surfaces, The Journal of Physical Chemistry C 115 (3) (2011) 736-743. doi:10. 1021/jp1097734.

[137] X. Zhang, H. Lhuissier, O. R. Enrquez, C. Sun, D. Lohse, Deactivation of microbubble nucleation sites by alcohol-water exchange, Langmuir 29 (32) (2013) 9979-9984. doi:10.1021/la402015q.

[138] M. Guan, W. Guo, L. Gao, Y. Tang, J. Hu, Y. Dong, Investigation on the temperature difference method for producing nanobubbles and their physical properties, ChemPhysChem 13 (8) (2012) 2115-2118. doi:10. 1002/cphc.201100912.

[139] W. Guo, H. Shan, M. Guan, L. Gao, M. Liu, Y. Dong, Investigation on nanobubbles on graphite substrate produced by the water- $\mathrm{NaCl}$ solution replacement, Surface Science 606 (17) (2012) 1462 - 1466. doi:10.1016/ j.susc.2012.05.018.

[140] M. Liu, W. Zhao, S. Wang, W. Guo, Y. Tang, Y. Dong, Study on nanobubble generation: Saline solution/water exchange method, ChemPhysChem 14 (11) (2013) 2589-2593. doi:10.1002/cphc.201201032.

[141] G. Shen, X. H. Zhang, Y. Ming, L. Zhang, Y. Zhang, J. Hu, Photocatalytic induction of nanobubbles on TiO2 surfaces, The Journal of Physical Chemistry C 112 (11) (2008) 4029-4032. doi:10.1021/jp711850d.

[142] S. Yang, P. Tsai, E. S. Kooij, A. Prosperetti, H. J. W. Zandvliet, D. Lohse, Electrolytically generated nanobubbles on highly orientated pyrolytic graphite surfaces, Langmuir 25 (3) (2009) 1466-1474. doi: $10.1021 / 1 \mathrm{a} 8027513$

[143] L. Zhang, Y. Zhang, X. Zhang, Z. Li, G. Shen, M. Ye, C. Fan, H. Fang, J. $\mathrm{Hu}$, Electrochemically controlled formation and growth of hydrogen nanobubbles, Langmuir 22 (19) (2006) 8109-8113. doi:10.1021/ la060859f. 
[144] Q. Chen, L. Luo, H. S. White, Electrochemical generation of a hydrogen bubble at a recessed platinum nanopore electrode, Langmuir 31 (15) (2015) 4573-4581. doi:10.1021/acs.langmuir.5b00234.

[145] L. Luo, H. S. White, Electrogeneration of single nanobubbles at sub-50nm-radius platinum nanodisk electrodes, Langmuir 29 (35) (2013) 1116911175. doi:10.1021/la402496z.

[146] Q. Chen, L. Luo, H. Faraji, S. W. Feldberg, H. S. White, Electrochemical measurements of single $\mathrm{H} 2$ nanobubble nucleation and stability at $\mathrm{Pt}$ nanoelectrodes, The Journal of Physical Chemistry Letters 5 (20) (2014) 3539-3544. doi:10.1021/jz501898r.

[147] Q. Chen, H. S. Wiedenroth, S. R. German, H. S. White, Electrochemical nucleation of stable $\mathrm{n} 2$ nanobubbles at pt nanoelectrodes, Journal of the American Chemical Society 137 (2015) 12064-12069. doi:10.1021/jacs. $5 b 07147$.

[148] S. R. German, Q. Chen, M. A. Edwards, H. S. White, Electrochemical measurement of hydrogen and nitrogen nanobubble lifetimes at pt nanoelectrodes, Journal of the Electrochemical Society 163 (2016) H3160H3166. doi:10.1149/2.0221604jes.

[149] S. R. German, M. A. Edwards, Q. Chen, H. S. White, Laplace pressure of individual h2 nanobubbles from pressure-addition electrochemistry, Nano Letters 16 (2016) 6691-6694. doi:10.1021/acs.nanolett.6b03590.

[150] H. Ren, S. R. German, M. A. Edwards, Q. Chen, H. S. White, Electrochemical generation of individual o2 nanobubbles via h2o2 oxidation, The Journal of Physical Chemistry Letters 8 (2017) 2450-2454. doi:10.1021/acs.jpclett.7b00882.

[151] Y. Liu, M. A. Edwards, S. R. German, Q. Chen, H. S. White, The dynamic steady state of an electrochemically generated nanobubble, Langmuir 33 (2017) 1845-1853. doi:10.1021/acs. langmuir.6b04607. 
[152] V. B. Svetovoy, R. G. P. Sanders, T. S. J. Lammerink, M. C. Elwenspoek, Combustion of hydrogen-oxygen mixture in electrochemically generated nanobubbles, Physical Review E 84 (2011) 035302. doi:10.1103/ PhysRevE.84.035302.

[153] V. B. Svetovoy, R. G. P. Sanders, K. Ma, M. C. Elwenspoek, New type of microengine using internal combustion of hydrogen and oxygen, Scientific Reports 4 (2014) 4296. doi:10.1038/srep04296

[154] S. Yang, E. S. Kooij, B. Poelsema, D. Lohse, H. J. W. Zandvliet, Correlation between geometry and nanobubble distribution on HOPG surface, EPL (Europhysics Letters) 81 (6) (2008) 64006. doi:10.1209/ $0295-5075 / 81 / 64006$.

[155] S. Michelin, E. Guérin, E. Lauga, Collective dissolution of microbubbles, Physical Review Fluids 3 (2018) 043601. doi:10.1103/PhysRevFluids. 3.043601 .

[156] B. Dollet, D. Lohse, Pinning stabilizes neighboring surface nanobubbles against ostwald ripening, Langmuir 32 (43) (2016) 11335-11339. doi: 10.1021/acs. langmuir.6b02136

[157] B. H. Tan, H. An, C.-D. Ohl, Resolving the pinning force of nanobubbles with optical microscopy, Physical Review Letters 118 (5) (2017) 054501. doi:10.1103/PhysRevLett.118.054501

[158] B. H. Tan, H. An, C.-D. Ohl, Surface nanobubbles are stabilized by hydrophobic attraction, Physical Review Letters 120 (16) (2018) 164502. doi:10.1103/PhysRevLett.120.164502

[159] Y. Wang, X. Li, S. Ren, H. T. Alem, L. Yang, D. Lohse, Entrapment of interfacial nanobubbles on nano-structured surfaces, Soft Matter (2017) 27-29doi:10.1039/c7sm01205e.

[160] Z.-L. Zou, N.-N. Quan, X.-Y. Wang, S. Wang, L.-M. Zhou, J. Hu, L.J. Zhang, Y.-M. Dong, The properties of surface nanobubbles formed on 
different substrates, Chinese Physics B 27 (2018) 086803. doi:10.1088/ $1674-1056 / 27 / 8 / 086803$.

[161] V. B. Svetovoy, I. Devi, J. H. Snoeijer, D. Lohse, Effect of disjoining pressure on surface nanobubbles, Langmuir 32 (43) (2016) 11188-11196. doi:10.1021/acs.langmuir.6b01812. 

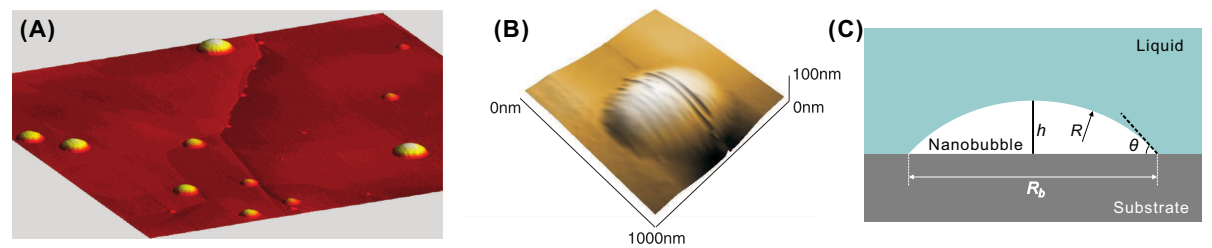

Figure 1: Surface nanobubbles. (A) Nanobubbles on an HOPG surface immersed in water. Reprinted with permission from Ref. 99]. Copyright 2010 American Chemical Society ; (B) Three-dimensional topological image of a single nanobubble on HOPG substrate at room temperature. Reprinted from Ref. [62]; (C) Schematic illustration of NB shape and parameters characterising its structure.
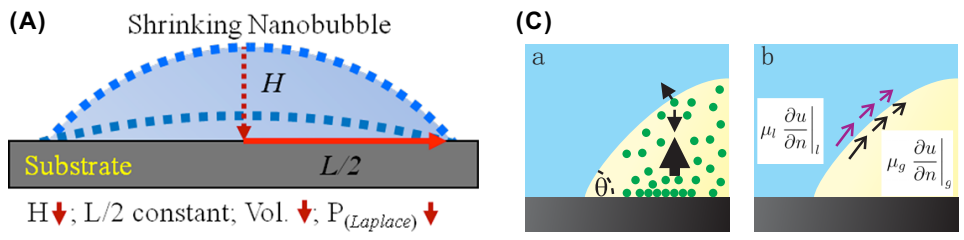

(B)
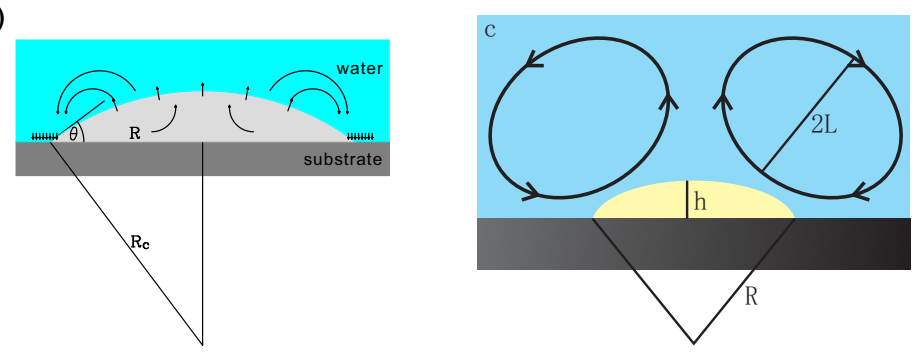

Figure 2: Schematic representation of main theories explaining the stability of nanobubbles. (A) Knudsen gas streaming. Upward flow of the Knudsen gas in the bulk, tangential component of the bulk Knudsen gas flow, and gas-rich liquid stream circulating around the bubble, which eventually transports the diffusive outfluxing gas back to the three-phase line for reentry. Reprinted with permission from Ref. 63]; (B) Dynamic equilibrium mechanism for surface NB stabilisation. Sketch of gas influx and outflux that leads to the stability of NB. Reprinted with permission from Ref. [51]; (C) Contact line pinning during NB growth and shrinkage. Reprinted with permission from Ref. [30]. Copyright 2013 American Chemical Society. 
(A)

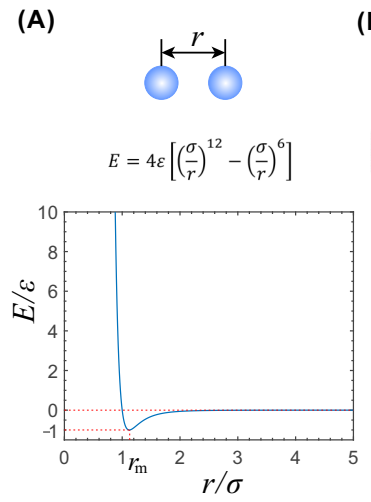

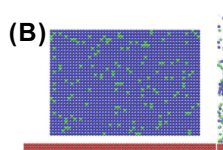

(a)

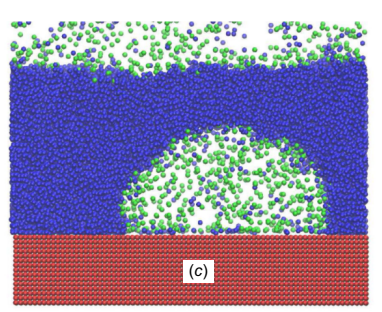

(C)

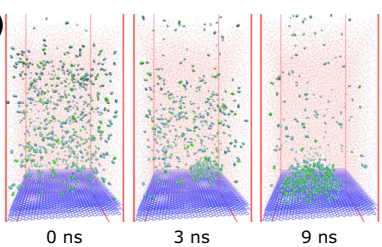

Water

Water

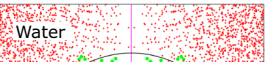

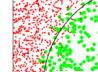

Surface nanobubble 3 .

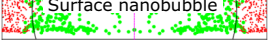

HOPG substrate

Figure 3: MD simulation based on LJ potentials (all-atom and CG models). (A) LJ potential as a function of distance $r$ between CG beads or atoms; (B) NB formation from MD simulation of a CG model. Reprinted with permission from Ref. 70; (C) NB formation from $\mathrm{N}_{2}$ and $\mathrm{O}_{2}$ atoms on an HOPG substrate immersed in water from all-atom MD simulation. Reprinted with permission from Ref. 68]. 


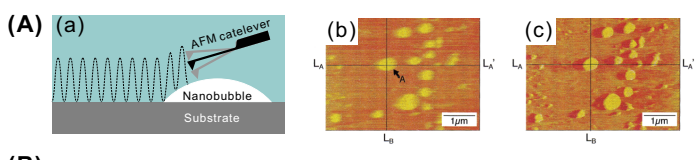

(B) (a)
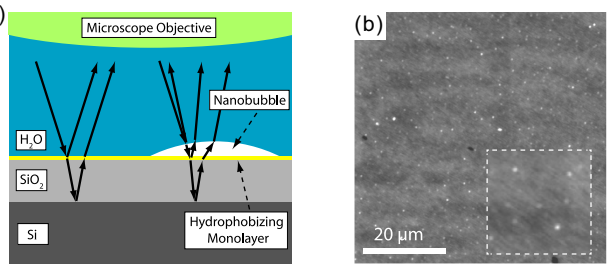

(C) (a)
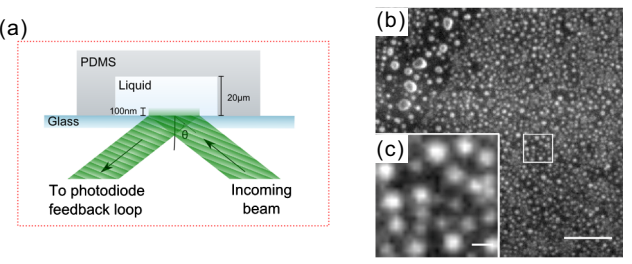

(D)

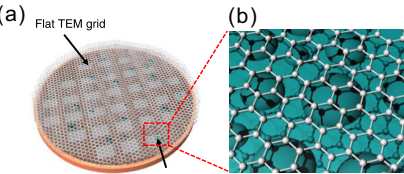

(c)

(e) $\begin{gathered}\text { Trapped water droplets } \\ \text { between graphene layers }\end{gathered}(\mathrm{f})$
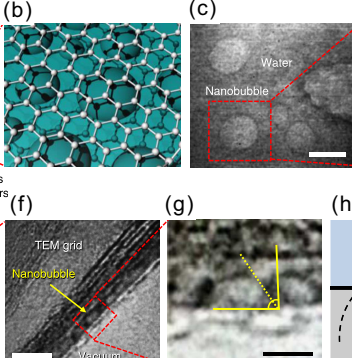

(d)

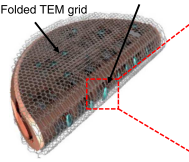

(g)

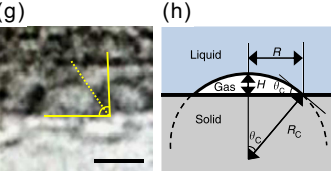

(E)
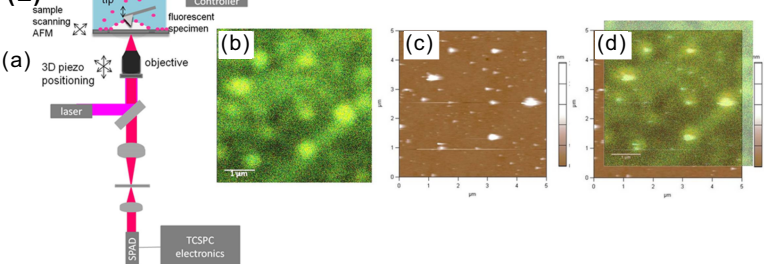

Figure 4: Main experimental methods for NBs imaging: (A) Schematic illustration of TMAFM and first AFM obtained images. Panels A(b-c) are reprinted with permission from Ref. [10]. Copyright 2000 American Chemical Society; (B) Schematic diagram of the nonintrusive optical interference-enhanced reflection microscopy technique and example of optical imaging by using this method. Reprinted with permission from Ref. [32; (C) Total-internalreflection-fluorescence (TIRF) microscopy for the study of NB dynamics. Schematic illustration of the TIRF microscopy for NB measurement and nanobubbles observed under TIRF microscopy. Reprinted with permission from Ref. 33; (D) Ultra-high vacuum TEM. Graphene liquid cell for TEM measurement and TEM images showing the morphologies of NBs in the graphene liquid cell. Reprinted with permission from Ref. 109]; (E) Schematic representation of fluorescence lifetime imaging microscopy and obtained results of surface nanobubbles nucleated by an ethanol-water exchange on OTS/glass. Reprinted with permission from Ref. 31. Copyright 2016 American Chemical Society. 

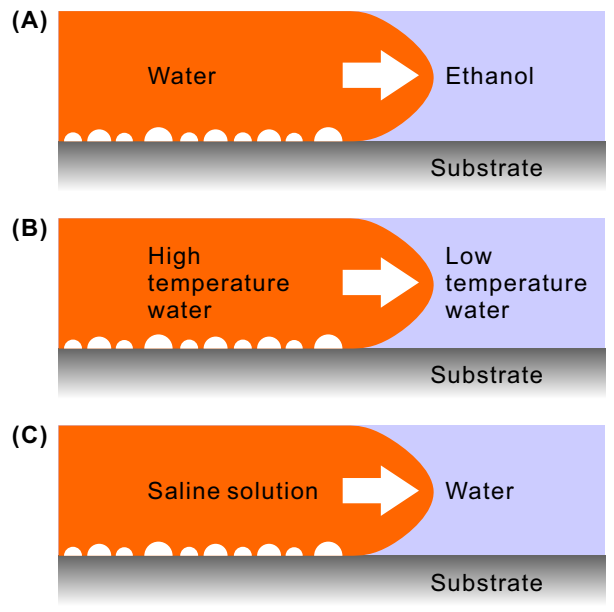

Figure 5: Formation of nanobubbles in experiment using the solvent exchange method. This can be achieved by using water-ethanol (A), temperature difference (B), and saline solution (C).
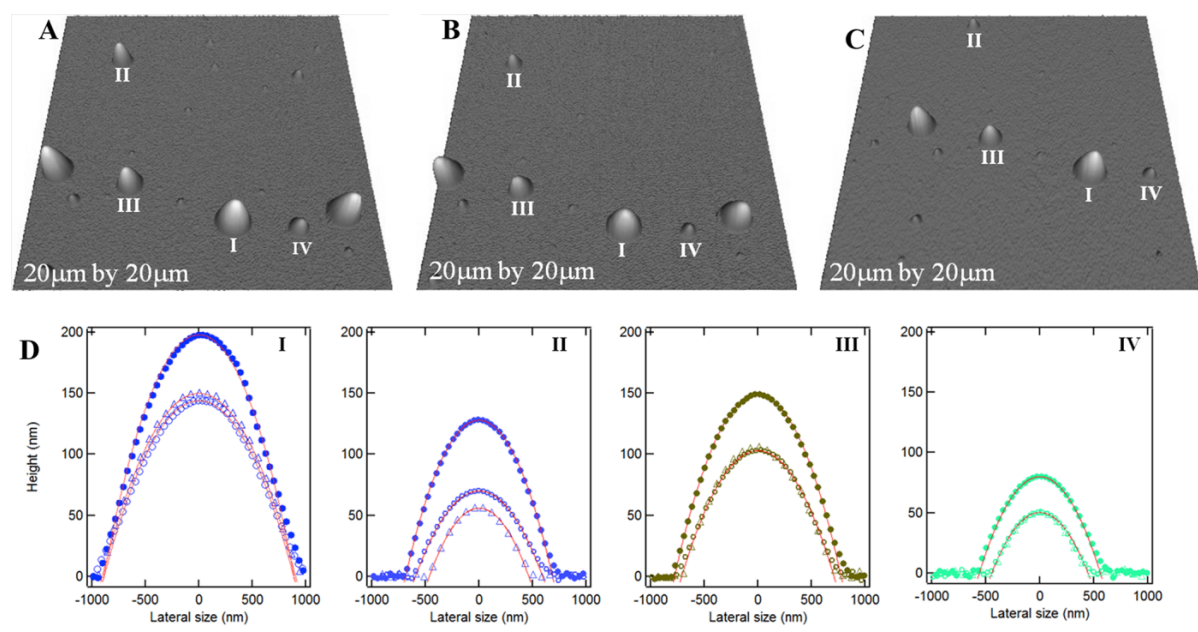

Figure 6: Contact line pinning. Morphology of surface NBs obtained from AFM and their fitting to spherical-cap shape. Reprinted with permission from Ref. 30. Copyright 2013 American Chemical Society. 